\title{
Abnormal Solvent Effects on Hydrogen Atom Abstraction. 3. Novel Kinetics in Sequential Proton Loss Electron Transfer (SPLET) Chemistry.
}

\author{
Grzegorz Litwinienko and K. U. Ingold \\ Warsaw University, Pasteura 1, 02-093 Warsaw, Poland \\ National Research Council, 100 Sussex Drive, Ottawa, Ontario, Canada, K1A 0R6
}

SUPPORTING MATERIAL

TABLE OF CONTENTS

Title

Table S1. Kinetic data for the reaction of dpph ${ }^{\bullet}$ with BIS in $n$-heptane.

Table S2. Kinetic data for the reaction of $\mathrm{dpph}^{\bullet}$ with $\mathrm{BIS}$ in $\mathrm{CCl}_{4}$ and methyl formate.

Table S3. Kinetic data for the reaction of $\mathrm{dpph}^{\bullet}$ with BIS in neat methanol.

Table S4. Kinetic data for the reaction of dpph with BIS in methanol containing $\mathrm{CH}_{3} \mathrm{CO}_{2} \mathrm{H}$.

Table S5. Kinetic data for the reaction of $\mathbf{d p p h}^{\bullet}$ with BIS in neat acetonitrile.

Table S6. Kinetic data for the reaction of $\mathbf{d p p h}^{\bullet}$ with BIS in acetonitrile containing $\mathrm{CH}_{3} \mathrm{CO}_{2} \mathrm{H}$.

Table S7. Kinetic data for the reaction of $\mathbf{d p p h}^{\bullet}$ with BIS in neat ethanol.

Table S8. Kinetic data for the reaction of dpph with BIS.in ethanol containing $\mathrm{CH}_{3} \mathrm{CO}_{2} \mathrm{H}$.

Table S9. Kinetic data for the reaction of $\mathrm{dpph}^{\bullet}$ with BIS in pivalonitrile and benzophenone.

Table S10. Kinetic data for the reaction of $\mathbf{d p p h}^{\bullet}$ with BIS in ethyl acetate.

Table S11. Kinetic data for the reaction of dpph with BIS in ethyl acetate containing $\mathrm{CH}_{3} \mathrm{CO}_{2} \mathrm{H}$.

Table S12. Kinetic data for the reaction of dpph $^{\bullet}$ with BIS in dioxane, 3-pentanone and acetophenone.

Table S13. Kinetic data for the reaction of $\mathbf{d p p h}^{\bullet}$ with BIS in $\gamma$-valeronitrile, pinacolone and THF.

Table S14. Kinetic data for the reaction of $\mathbf{d p p h}^{\bullet}$ with BIS in acetone and acetone containing $\mathrm{CH}_{3} \mathrm{CO}_{2} \mathrm{H}$.

Table S15. Kinetic data for the reaction of $\mathrm{dpph}^{\bullet}$ with BIS in cyclohexanone and di-cyclopropylketone.

Table S16. Kinetic data for the reaction of $\mathrm{dpph}^{\bullet}$ with BIS in benzonitrile.

Table S17. Kinetic data for the reaction of $\mathbf{d p p h}^{\bullet}$ with BIS in benzonitrile containing $\mathrm{CH}_{3} \mathrm{CO}_{2} \mathrm{H}$.

Table S18. Kinetic data for the reaction of $\mathbf{d p p h}^{\bullet}$ with BIS in DMSO.

Table S19. Kinetic data for the reaction of dpph with BIS in DMSO containing $\mathrm{CH}_{3} \mathrm{CO}_{2} \mathrm{H}$.

Table S20. Kinetic data for the reaction of dpph $^{\bullet}$ with 2-tert-butyl-4,6-dimethylphenol in $n$-heptane, 


\section{TABLE OF CONTENTS continued...}

Title

Page

Table S21. Kinetic data for the reaction of $\mathbf{d p p h}^{\bullet}$ with 2,4,6-trimethylphenol in fourteen solvents

Table S22. Parameters used for calculation of the equilibrium constant $K$ for HB complex formation between BIS and DMSO.

Table S23. Parameters used for calculation of the equilibrium constant $K$ for HB complex formation between BIS and acetonitrile.

Table S24. Values $K, \log K_{A}^{H}$, and $\alpha_{2}^{H}$ for BIS calculated from IR measurements of HB complex formation with DMSO and acetonitrile.

Table S25. Parameters used for calculation of the equilibrium constant $K$ for HB complex formation between 2-tert-butyl-4,6-dimethylphenol and DMSO.

Figure S1. Plots of dpph decay in reaction with BIS in acetonitrile and acidified acetonitrile.

Figure S2. Plot of $\log k^{S}$ for 2,4,6-trimethylphenol/dpph ${ }^{\bullet}$ reaction in several solvents.

Figure S3. Plots of $[\mathrm{ArOH}]_{\mathrm{o}} /[\mathrm{ArOH}]_{\text {free }}$ vs. $[\mathrm{DMSO}]_{\mathrm{free}}$ for BIS.

Figure S4. Plots of $[\mathrm{ArOH}]_{\mathrm{o}} /[\mathrm{ArOH}]_{\text {free }}$ vs. [acetonitrile $]_{\text {free }}$ for BIS.

Figure S5. First order plots of decay of $\mathbf{d p p h}^{\bullet}$ reacting with 2-tert-butyl-4,6-dimethylphenol in acetone.

Figure S6. IR spectra of 2-tert-butyl-4,6-dimethylphenol (22.4 mM) in $\mathrm{CCl}_{4}$ containing DMSO at different concentrations.

Figure S7. Plots of $[\mathrm{ArOH}]_{\mathrm{o}} /[\mathrm{ArOH}]_{\text {free }}$ vs. $[\mathrm{DMSO}]_{\text {free }}$ for 2-tert-butyl-4,6-dimethylphenol.

Figure S8. Kinetic traces of dpph $^{\bullet}$ decay in neat DMSO and in DMSO containing acetic acid.

Figure S9. Zero-order plots of $\mathbf{d p p h}^{\bullet}$ decay in reaction with BIS in acetone - determination of the rate of ionization

Figure S10. Zero-order plots of $\mathbf{d p p h}^{\bullet}$ decay in its reaction with BIS in acetonitrile. 
Table S1. Kinetic data for the reaction of $\mathrm{dpph}^{\bullet}$ with BIS in $n$-heptane. Concentration of BIS, [BIS], pseudo-first-order rate constant, $\mathrm{k}_{\mathrm{ex}}, \mathrm{R}^{2}$ and bimolecular rate constant, $\boldsymbol{k}^{\boldsymbol{s}}$, are lieted for each data set. The calculated mean values of $\boldsymbol{k}^{\boldsymbol{S}}$ are listed with absolute errors.

\begin{tabular}{|c|c|c|c|}
\hline $\begin{array}{l}{[\mathrm{BIS}]} \\
/ \mathrm{mM}\end{array}$ & $\begin{array}{c}10^{3} \times k_{e x} \\
/ s^{-1}\end{array}$ & $\begin{array}{l}\text { [BIS] } \\
/ \mathrm{mM}\end{array}$ & $\begin{array}{c}10^{3} \times \mathrm{k}_{\mathrm{ex}} \\
/ \mathrm{s}^{-1}\end{array}$ \\
\hline 2.8 & 157.7 & 1.04 & 61.4 \\
\hline 1.9 & 104.6 & 0.93 & 55.1 \\
\hline 1.2 & 72.2 & 0.72 & 42.2 \\
\hline 0.8 & 48.6 & 0.56 & 33.2 \\
\hline 0.5 & 28.6 & 0.44 & 25.5 \\
\hline 0.3 & 17.0 & 0.33 & 19.1 \\
\hline \multicolumn{2}{|c|}{$\mathbf{k}=55$} & \multicolumn{2}{|c|}{$\mathbf{k}=60$} \\
\hline \multicolumn{2}{|c|}{$\Delta \mathbf{k}^{a}=0.77$} & \multicolumn{2}{|c|}{$\begin{aligned} \Delta \mathbf{k}^{a} & =0.51 \\
\mathbf{R}^{2} & =0.9999\end{aligned}$} \\
\hline
\end{tabular}

${ }^{a}$ Calculated as the confidence interval of the slope for the $90 \%$ confidence level.

$k^{\text {heptane }}=57 \pm 3 \mathrm{M}^{-1} \mathrm{~s}^{-1}$

Table S2. Kinetic data for the reaction of $\mathrm{dpph}^{\bullet}$ with BIS in carbon tetrachloride and methyl formate. Symbols the same as for Table 1.

\begin{tabular}{|c|c|c|c|}
\hline \multicolumn{2}{|c|}{$\mathrm{CCl}_{4}$} & \multicolumn{2}{|c|}{ Methyl formate } \\
\hline $\begin{array}{l}{[\mathrm{BIS}]} \\
/ \mathrm{mM}\end{array}$ & $\begin{array}{c}10^{3} \times \mathrm{k}_{\mathrm{ex}} \\
/ \mathrm{s}^{-1}\end{array}$ & $\begin{array}{l}{[\mathrm{BIS}]} \\
/ \mathrm{mM}\end{array}$ & $\begin{array}{c}10^{3} \times \mathrm{k}_{\mathrm{e}} \\
/ \mathrm{s}^{-1}\end{array}$ \\
\hline 1.44 & 34.5 & 2.16 & 2.67 \\
\hline 1.09 & 28.6 & 1.92 & 2.46 \\
\hline 0.82 & 21.0 & 1.50 & 1.97 \\
\hline 0.58 & 15.5 & 1.13 & 1.67 \\
\hline 0.39 & 10.3 & 0.80 & 1.32 \\
\hline 0.20 & 5.4 & 0.46 & 1.05 \\
\hline \multicolumn{2}{|c|}{$\begin{aligned} \Delta \mathbf{k}^{a} & =1.8 \\
\mathbf{R}^{2} & =0.9962\end{aligned}$} & \multicolumn{2}{|c|}{$\begin{aligned} \Delta \mathbf{k}^{a} & =0.05 \\
\mathbf{R}^{2} & =0.9972\end{aligned}$} \\
\hline
\end{tabular}

${ }^{a}$ Clculated as the confidence interval of the slope for the $90 \%$ confidence level.

$k^{\mathrm{CCl} 4}=25 \pm 2 \mathrm{M}^{-1} \mathrm{~s}^{-1}$

$k^{\text {methyl formate }}=1.0 \pm 0.1 \mathrm{M}^{-1} \mathrm{~s}^{-1}$ 
Table S3. Kinetic data for the reaction of $\mathbf{d p p h}^{\bullet}$ with BIS in methanol. Symbols are the same as described in Table S1.

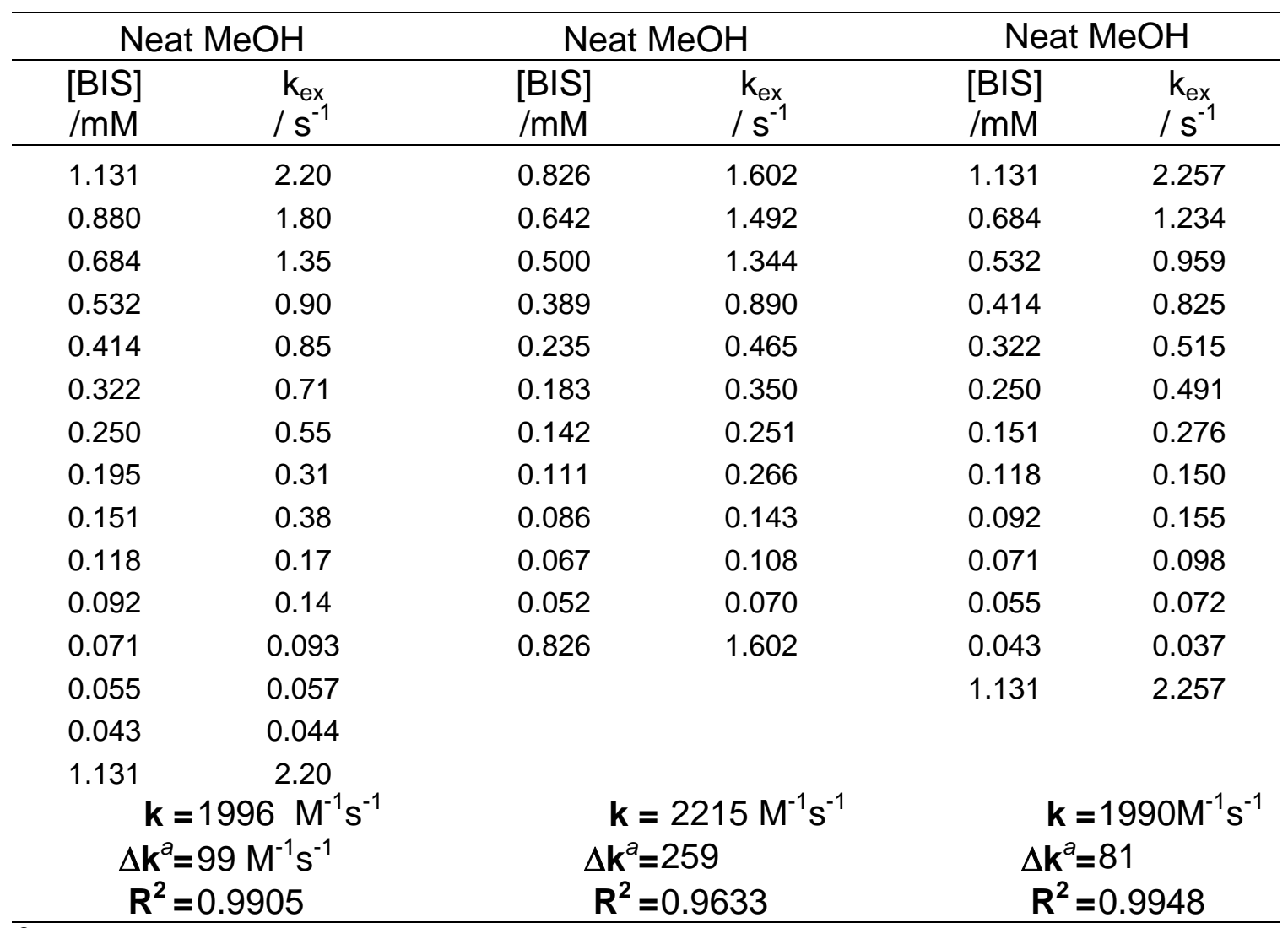

${ }^{a}$ Caculated as the confidence interval of the slope for the $90 \%$ confidence level.

$k^{\text {methanol }}=2100 \pm 100 \mathrm{M}^{-1} \mathrm{~s}^{-1}$ 
Table S4. Kinetic data for the reaction of $\mathbf{d p p h}^{\bullet}$ with BIS in acidified methanol. Symbols are the same as described in Table S1.

\begin{tabular}{|c|c|c|c|c|c|c|c|}
\hline \multicolumn{3}{|c|}{$\mathrm{MeOH} / 10 \mathrm{mM}$ acetic } & \multicolumn{2}{|c|}{ MeOH / 10 mM acetic } & & \multicolumn{2}{|c|}{ MeOH / 10 mM acetic } \\
\hline $\begin{array}{l}\text { [BIS] } \\
/ \mathrm{mM} \\
\end{array}$ & \multicolumn{2}{|l|}{$\begin{array}{c}\mathrm{k}_{\mathrm{ex}} \times 10^{3} \\
/ \mathrm{s}^{-1}\end{array}$} & $\begin{array}{l}{[\mathrm{BIS}]} \\
/ \mathrm{mM}\end{array}$ & \multicolumn{2}{|l|}{$\begin{array}{c}\mathrm{k}_{\mathrm{e} \times 10^{3}} \\
/ \mathrm{s}^{-1}\end{array}$} & $\begin{array}{l}\text { [BIS] } \\
\text { /mM } \\
\end{array}$ & $\begin{array}{l}\mathrm{k}_{\mathrm{ex}} \times 10^{3} \\
/ \mathrm{s}^{-1}\end{array}$ \\
\hline 0.808 & 7.00 & & 8.012 & 69.41 & & 0.500 & 5.79 \\
\hline 0.538 & 4.36 & & 5.341 & 55.61 & & 0.389 & 4.96 \\
\hline 0.359 & 3.29 & & 2.374 & 27.08 & & 0.302 & 4.28 \\
\hline 0.239 & 2.00 & & 1.583 & 18.46 & & 0.235 & 3.32 \\
\hline 0.160 & 1.48 & & 1.055 & 12.20 & & 0.183 & 2.77 \\
\hline 0.106 & 1.08 & & 0.703 & 8.94 & & 0.142 & 2.28 \\
\hline 0.071 & 0.755 & & 0.469 & 6.24 & & 0.111 & 1.86 \\
\hline 0.047 & 0.566 & & 0.313 & 4.17 & & & \\
\hline \multirow[t]{4}{*}{0.032} & 0.535 & & 0.208 & 3.73 & & & \\
\hline & & & 0.139 & 2.70 & & & \\
\hline & & & 0.093 & 1.46 & & & \\
\hline & & & 0.062 & 1.15 & & & \\
\hline \multicolumn{3}{|c|}{$\begin{aligned} \mathbf{k} & =8.3 \\
\Delta \mathbf{k}^{a} & =0.4 \\
\mathbf{R}^{2} & =0.9961\end{aligned}$} & \multicolumn{2}{|c|}{$\begin{aligned} \mathbf{k} & =9.0 \\
\Delta \mathbf{k}^{\mathrm{a}} & =0.9 \\
\mathbf{R}^{2} & =0.9865\end{aligned}$} & \multicolumn{3}{|c|}{$\begin{aligned} \mathbf{k} & =10.0 \\
\Delta \mathbf{k}^{a} & =1.0 \\
\mathbf{R}^{2} & =0.9863\end{aligned}$} \\
\hline \multicolumn{2}{|c|}{$\begin{array}{l}\mathrm{MeOH} / \\
100 \mathrm{mM} \text { acetic }\end{array}$} & \multicolumn{2}{|c|}{$\begin{array}{l}\mathrm{MeOH} / \\
100 \mathrm{mM} \text { acetic }\end{array}$} & $\begin{array}{l}\mathrm{MeOH} / \\
100 \mathrm{mM} \text { ac }\end{array}$ & cetic & $\begin{array}{l}\mathrm{MeOH} / \\
1000 \mathrm{mM}\end{array}$ & acetic \\
\hline $\begin{array}{l}\text { [BIS] } \\
/ \mathrm{mM}\end{array}$ & $\begin{array}{l}\mathrm{k}_{\mathrm{ex}} \times 10^{3} \\
/ \mathrm{s}^{-1}\end{array}$ & $\begin{array}{l}\mathrm{BIS}] \\
/ \mathrm{mM} \\
\end{array}$ & $\begin{array}{c}\mathrm{k}_{\mathrm{ex}} \times 10^{3} \\
/ \mathrm{s}^{-1}\end{array}$ & $\begin{array}{l}\mathrm{BIS}] \\
/ \mathrm{mM}\end{array}$ & $\begin{array}{c}\mathrm{k}_{\mathrm{ex}} \times 10^{3} \\
/ \mathrm{s}^{-1}\end{array}$ & $\begin{array}{c}\text { [BIS] } \\
/ \mathrm{mM} \\
\end{array}$ & $\begin{array}{c}\mathrm{k}_{\mathrm{ex}} \times 10^{3} \\
/ \mathrm{s}^{-1}\end{array}$ \\
\hline 0.642 & 2.470 & 0.538 & 2.255 & 2.374 & 9.275 & 1.062 & 2.330 \\
\hline 0.500 & 2.220 & 0.359 & 1.860 & 1.583 & 7.420 & 0.826 & 2.090 \\
\hline 0.389 & 1.780 & 0.239 & 1.470 & 1.055 & 4.165 & 0.642 & 1.900 \\
\hline 0.302 & 1.490 & 0.160 & 1.230 & 0.703 & 3.230 & 0.500 & 1.690 \\
\hline 0.235 & 1.310 & 0.106 & 0.842 & 0.469 & 2.360 & 0.389 & 1.540 \\
\hline 0.183 & 1.100 & 0.071 & 0.664 & 0.313 & 1.770 & 0.302 & 1.380 \\
\hline 0.142 & 1.010 & 0.047 & 0.600 & 0.208 & 1.190 & 0.235 & 1.220 \\
\hline \multirow[t]{4}{*}{0.111} & 0.777 & 0.032 & 0.473 & 0.139 & 0.976 & 0.183 & 1.120 \\
\hline & & & & 0.093 & 0.852 & 0.142 & 0.998 \\
\hline & & & & 0.062 & 0.665 & & \\
\hline & & & & 0.041 & 0.559 & & \\
\hline \multicolumn{2}{|c|}{$\begin{aligned} \mathbf{k} & =3.2 \\
\Delta \mathbf{k}^{a} & =0.3 \\
\mathbf{R}^{2} & =0.9865\end{aligned}$} & \multicolumn{2}{|c|}{$\begin{aligned} \mathbf{k} & =3.6 \\
\Delta \mathbf{k}^{a} & =0.5 \\
\mathbf{R}^{2} & =0.9644\end{aligned}$} & \multicolumn{2}{|c|}{$\begin{aligned} \mathbf{k} & =3.9 \\
\Delta \mathbf{k}^{a} & =0.3 \\
\mathbf{R}^{2} & =0.9882\end{aligned}$} & \multicolumn{2}{|c|}{$\begin{aligned} \mathbf{k} & =1.4 \\
\Delta \mathbf{k}^{a} & =0.2 \\
\mathbf{R}^{2} & =0.9744\end{aligned}$} \\
\hline \multicolumn{8}{|c|}{$\begin{array}{l}{ }^{a} \text { Caculated as the confidence interval of the slop } \\
k^{M e O H / C H 3 C O 2 H}=9.1 \pm 1.0 \mathrm{M}^{-1} \mathrm{~s}^{-1} \\
k^{\mathrm{MeOH} / 100 \mathrm{mM} \mathrm{CH} 3 \mathrm{CO} 2 \mathrm{H}}=3.6 \pm 0.4 \mathrm{M}^{-1} \mathrm{~s}^{-1} \\
k^{\mathrm{MeOH} / 1000 \mathrm{mM} \mathrm{CH} 3 \mathrm{CO}_{2} \mathrm{H}}=1.4 \pm 0.2 \mathrm{M}^{-1} \mathrm{~s}^{-1}\end{array}$} \\
\hline
\end{tabular}


Table S5. Kinetic data for the reaction of $\mathbf{d p p h}^{\bullet}$ with BIS in acetonitrile. Symbols are the same as described in Table S1.

\begin{tabular}{|c|c|c|c|c|c|}
\hline \multicolumn{2}{|c|}{ Neat acetonitrile } & \multicolumn{2}{|c|}{ Neat acetonitrile } & \multicolumn{2}{|c|}{ Neat acetonitrile } \\
\hline $\begin{array}{l}\text { [BIS] } \\
/ \mathrm{mM}\end{array}$ & $\begin{array}{c}\mathrm{k}_{\mathrm{ex}} \\
/ \mathrm{s}^{-1}\end{array}$ & $\begin{array}{l}\text { [BIS] } \\
/ \mathrm{mM}\end{array}$ & $\begin{array}{c}\mathrm{k}_{\mathrm{ex}} \\
/ \mathrm{s}^{-1}\end{array}$ & $\begin{array}{l}\text { [BIS] } \\
/ \mathrm{mM}\end{array}$ & $\begin{array}{c}\mathrm{k}_{\mathrm{ex}} \\
/ \mathrm{s}^{-1} \\
\end{array}$ \\
\hline 0.474 & 6.80 & 0.902 & 12.51 & 0.369 & 6.44 \\
\hline 0.369 & 5.11 & 0.750 & 10.68 & 0.287 & 4.27 \\
\hline 0.287 & 3.46 & 0.620 & 8.96 & 0.223 & 3.15 \\
\hline 0.223 & 3.48 & 0.440 & 5.91 & 0.174 & 2.46 \\
\hline 0.135 & 1.80 & 0.315 & 3.91 & 0.135 & 1.53 \\
\hline 0.105 & 1.77 & 0.225 & 2.54 & 0.105 & 1.32 \\
\hline 0.082 & 1.15 & & & 0.064 & 0.74 \\
\hline 0.064 & 1.03 & & & \multirow{2}{*}{\multicolumn{2}{|c|}{ k =17866 }} \\
\hline \multicolumn{2}{|c|}{$\mathbf{k}=13641$} & \multicolumn{2}{|c|}{$k=14956$} & & \\
\hline \multicolumn{2}{|c|}{$\Delta \mathbf{k}^{a}=1214$} & \multicolumn{2}{|c|}{$\mathbf{R}^{2}=0.9973$} & $\Delta \mathbf{k}^{a}=1853$ & $\mathbf{R}^{2}=0.9817$ \\
\hline
\end{tabular}

${ }^{a}$ Caculated as the confidence interval of the slope for the $90 \%$ confidence level.

$$
k^{\text {acetonitrile }}=(15 \pm 3) \times 10^{3} \mathrm{M}^{-1} \mathrm{~s}^{-1}
$$

Table S6. Kinetic data for the reaction of $\mathbf{d p p h}^{\bullet}$ with BIS in acidified acetonitrile. Symbols are the same as described in Table S1.

\begin{tabular}{|c|c|c|c|c|c|c|c|}
\hline \multicolumn{2}{|c|}{$\begin{array}{l}\text { acetonitrile / } \\
10 \mathrm{mM} \text { acetic }\end{array}$} & \multicolumn{2}{|c|}{$\begin{array}{l}\text { acetonitrile / } \\
10 \mathrm{mM} \text { acetic }\end{array}$} & \multicolumn{2}{|c|}{$\begin{array}{l}\text { acetonitrile / } \\
100 \mathrm{mM} \text { acetic }\end{array}$} & \multicolumn{2}{|c|}{$\begin{array}{l}\text { acetonitrile / } \\
100 \mathrm{mM} \text { acetic }\end{array}$} \\
\hline $\begin{array}{l}\text { [BIS] } \\
/ \mathrm{mM}\end{array}$ & $\begin{array}{l}\mathrm{k}_{\mathrm{ex}} \\
/ \mathrm{s}^{-1}\end{array}$ & $\begin{array}{l}\text { [BIS] } \\
/ \mathrm{mM}\end{array}$ & $\begin{array}{l}k_{e x} \\
/ s^{-1}\end{array}$ & $\begin{array}{l}\text { [BIS] } \\
/ \mathrm{mM}\end{array}$ & $\begin{array}{l}\mathrm{k}_{\mathrm{ex}} \\
/ \mathrm{s}^{-1}\end{array}$ & $\begin{array}{l}\text { [BIS] } \\
/ \mathrm{mM}\end{array}$ & $\begin{array}{l}k_{\mathrm{ex}} \\
/ \mathrm{s}^{-1}\end{array}$ \\
\hline 4.12 & 22.250 & 1.21 & 7.575 & 0.970 & 0.247 & 0.552 & 0.134 \\
\hline 1.60 & 18.135 & 0.941 & 6.013 & 0.754 & 0.207 & 0.429 & 0.087 \\
\hline 1.25 & 13.830 & 0.732 & 4.286 & 0.587 & 0.180 & 0.334 & 0.068 \\
\hline 0.970 & 12.055 & 0.443 & 2.601 & 0.456 & 0.134 & 0.260 & 0.052 \\
\hline 0.754 & 8.583 & 0.345 & 2.299 & 0.355 & 0.086 & 0.202 & 0.035 \\
\hline 0.587 & 6.537 & 0.268 & 1.641 & 0.276 & 0.060 & 0.157 & 0.031 \\
\hline 0.456 & 5.134 & 0.208 & 1.866 & 0.215 & 0.050 & 0.122 & 0.018 \\
\hline 0.355 & 3.366 & 0.162 & 1.373 & 0.167 & 0.042 & 0.095 & 0.014 \\
\hline 0.276 & 2.001 & 0.126 & 1.336 & 0.130 & 0.041 & 0.074 & 0.011 \\
\hline 0.215 & 2.311 & & & 0.101 & 0.024 & & \\
\hline 0.167 & 2.218 & & & 0.079 & 0.017 & & \\
\hline 0.130 & 1.137 & & & & & & \\
\hline 0.101 & 1.050 & & & & & & \\
\hline 0.079 & 0.759 & \multirow{2}{*}{\multicolumn{2}{|c|}{$k=5832$}} & \multirow{2}{*}{\multicolumn{2}{|c|}{$k=273$}} & \multirow{2}{*}{\multicolumn{2}{|c|}{274}} \\
\hline$k=$ & 5602 & & & & & & \\
\hline$\Delta \mathbf{k}^{a}=$ & 224 & \multirow{2}{*}{\multicolumn{2}{|c|}{$\begin{aligned} \Delta \mathbf{k}^{a} & =460 \\
\mathbf{R}^{2} & =0.9872\end{aligned}$}} & \multirow{2}{*}{\multicolumn{2}{|c|}{$\begin{aligned} \Delta \mathbf{k}^{a} & =29 \\
\mathbf{R}^{2} & =0.9813\end{aligned}$}} & \multirow{2}{*}{\multicolumn{2}{|c|}{$\begin{aligned} \Delta \mathbf{k}^{a} & =22 \\
\mathbf{R}^{2} & =0.9843\end{aligned}$}} \\
\hline $\mathbf{R}^{2}$ & 0.9938 & & & & & & \\
\hline
\end{tabular}


Table S6. continued

\begin{tabular}{|c|c|c|c|}
\hline \multicolumn{2}{|c|}{$\begin{array}{c}\text { acetonitrile / } \\
1000 \mathrm{mM} \text { acetic }\end{array}$} & \multicolumn{2}{|c|}{$\begin{array}{c}\text { acetonitrile / } \\
1000 \mathrm{mM} \text { acetic }\end{array}$} \\
\hline $\begin{array}{l}\text { [BIS] } \\
/ \mathrm{mM}\end{array}$ & $\begin{array}{c}\mathrm{k}_{\mathrm{ex}} \times 10^{3} \\
/ \mathrm{s}^{-1}\end{array}$ & $\begin{array}{l}\text { [BIS] } \\
/ \mathrm{mM}\end{array}$ & $\begin{array}{c}\mathrm{k}_{\mathrm{e} \times} \times 10^{3} \\
/ \mathrm{s}^{-1}\end{array}$ \\
\hline 2.06 & 6.28 & 1.45 & 4.57 \\
\hline 1.60 & 5.49 & 1.13 & 3.29 \\
\hline 1.25 & 4.98 & 0.879 & 2.67 \\
\hline 0.970 & 4.61 & 0.683 & 2.42 \\
\hline 0.754 & 3.95 & 0.532 & 2.43 \\
\hline \multirow[t]{3}{*}{0.587} & 3.83 & 0.322 & 2.12 \\
\hline & & 0.195 & 1.51 \\
\hline & & 0.118 & 1.20 \\
\hline $\begin{array}{r}\mathbf{k} \\
\Delta \mathbf{k}^{\mathrm{c}} \\
\mathbf{R}^{2}\end{array}$ & $\begin{array}{l}7 \\
2 \\
9892\end{array}$ & $\Delta$ & $\begin{array}{l}2 \\
4 \\
9398\end{array}$ \\
\hline \multicolumn{4}{|c|}{ aculated as the confidence interval of the slope for the $90 \%$ confidence level. } \\
\hline \multicolumn{4}{|c|}{ 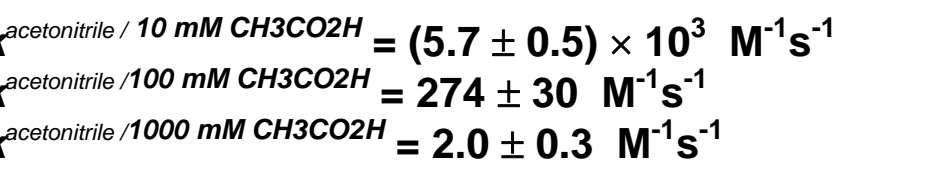 } \\
\hline
\end{tabular}

Table S7. Kinetic data for the reaction of $\mathrm{dpph}^{\bullet}$ with BIS in ethanol. Symbols are the same as described in Table S1.

\begin{tabular}{|c|c|c|c|c|c|}
\hline \multicolumn{2}{|c|}{ neat ethanol } & \multicolumn{2}{|c|}{ neat ethanol } & \multicolumn{2}{|c|}{ neat ethanol } \\
\hline $\begin{array}{l}\text { [BIS] } \\
/ \mathrm{mM}\end{array}$ & $\begin{array}{c}\mathrm{k}_{\mathrm{ex}} \\
/ \mathrm{s}^{-1}\end{array}$ & $\begin{array}{l}\text { [BIS] } \\
/ \mathrm{mM}\end{array}$ & $\begin{array}{c}\mathrm{k}_{\mathrm{ex}} \\
/ \mathrm{s}^{-1}\end{array}$ & $\begin{array}{l}\text { [BIS] } \\
/ \mathrm{mM}\end{array}$ & $\begin{array}{l}\mathrm{k}_{\mathrm{ex}} \\
/ \mathrm{s}^{-1}\end{array}$ \\
\hline 0.809 & 0.947 & 0.830 & 0.944 & 1.07 & 1.820 \\
\hline 0.629 & 0.756 & 0.645 & 0.608 & 0.830 & 1.250 \\
\hline 0.490 & 0.466 & 0.502 & 0.404 & 0.645 & 0.847 \\
\hline 0.296 & 0.290 & 0.390 & 0.310 & 0.502 & 0.674 \\
\hline 0.139 & 0.191 & 0.236 & 0.270 & 0.390 & 0.665 \\
\hline 0.108 & 0.083 & 0.184 & 0.161 & 0.236 & 0.490 \\
\hline 0.084 & 0.041 & 0.143 & 0.150 & 0.184 & 0.279 \\
\hline 0.066 & 0.027 & 0.111 & 0.105 & 0.143 & 0.233 \\
\hline & & 0.086 & 0.079 & 0.111 & 0.139 \\
\hline & & 0.067 & 0.043 & 0.086 & 0.121 \\
\hline$k=$ & & $\begin{array}{c}0.052 \\
\mathbf{k}=1\end{array}$ & 0 & $\begin{array}{r}0.067 \\
\mathbf{k}=1\end{array}$ & 0.109 \\
\hline $\begin{array}{r}\Delta k^{a}= \\
R^{2}=\end{array}$ & 336 & $\begin{aligned} \Delta \mathbf{k}^{a} & =1 \\
\mathbf{R}^{2} & =0\end{aligned}$ & 619 & $\begin{aligned} \Delta \mathbf{k}^{a} & =1 \\
\mathbf{R}^{2} & =0\end{aligned}$ & 2 \\
\hline
\end{tabular}


Table S8. Kinetic data for the reaction of dpph $^{\bullet}$ with BIS in acidified ethanol. Symbols are the same as described in Table S1.

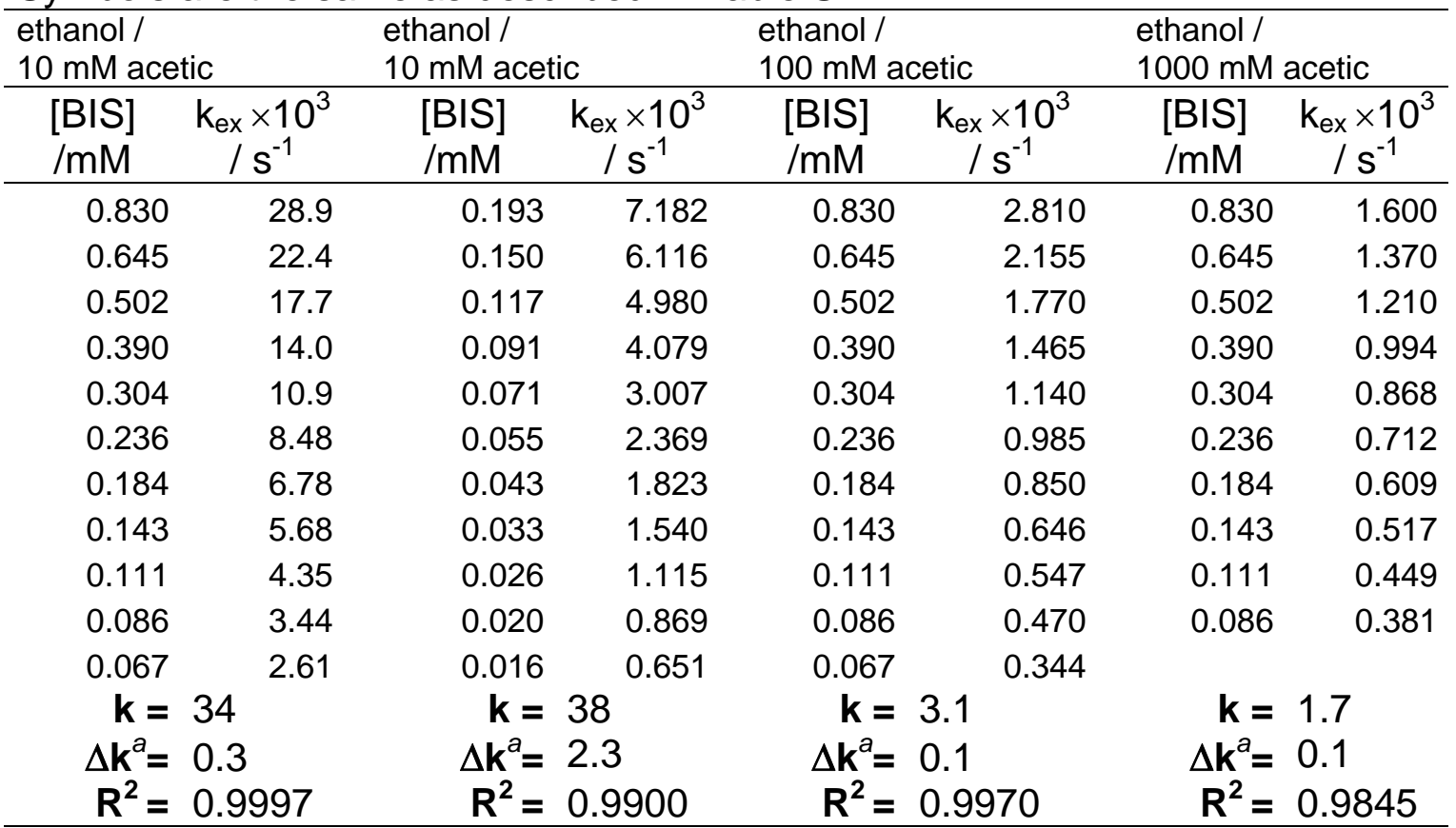

${ }^{a}$ Caculated as the confidence interval of the slope for the $90 \%$ confidence level.

$k^{\text {ethanol/10 mM CH3CO2H }}=36 \pm 2 \quad M^{-1} s^{-1}$
$k^{\text {ethanol/100 mM CH3CO2H }}=3.1 \pm 0.1 \mathrm{M}^{-1} \mathrm{~s}^{-1}$
$k^{\text {ethanol/1000 mM CH3CO2H }}=1.7 \pm 0.1 \mathrm{M}^{-1} \mathrm{~s}^{-1}$

Table S9. Kinetic data for the reaction of $\mathbf{d p p h}^{\bullet}$ with BIS in pivalonitrile and in $1 \mathrm{M}$ benzophenone in benzene. Symbols are the same as described in Table S1.

\begin{tabular}{|c|c|c|c|c|c|}
\hline \multicolumn{2}{|c|}{ pivalonitrile } & \multicolumn{2}{|c|}{ pivalonitrile } & \multicolumn{2}{|c|}{$\mathrm{Ph}_{2} \mathrm{CO} / \mathrm{PhH}$} \\
\hline $\begin{array}{l}\text { [BIS] } \\
/ \mathrm{mM}\end{array}$ & $\begin{array}{c}\mathrm{k}_{\mathrm{ex}} \times 10^{3} \\
/ \mathrm{s}^{-1}\end{array}$ & $\begin{array}{l}\text { [BIS] } \\
/ \mathrm{mM}\end{array}$ & $\begin{array}{c}\mathrm{k}_{\mathrm{ex}} \times 10^{3} \\
/ \mathrm{s}^{-1}\end{array}$ & $\begin{array}{l}\text { [BIS] } \\
/ \mathrm{mM}\end{array}$ & $\begin{array}{c}\mathrm{k}_{\mathrm{ex}} \times 10^{3} \\
/ \mathrm{s}^{-1}\end{array}$ \\
\hline 2.068 & 30.840 & 1.920 & 22.73 & 2.749 & 24.90 \\
\hline 1.571 & 21.945 & 1.370 & 14.85 & 2.330 & 20.15 \\
\hline 1.200 & 20.475 & 0.980 & 9.71 & 1.800 & 15.95 \\
\hline 0.800 & 14.925 & 0.730 & 6.90 & 1.350 & 12.08 \\
\hline 0.560 & 11.630 & 0.550 & 5.60 & 6.750 & 49.40 \\
\hline \multirow[t]{3}{*}{0.280} & 6.595 & & & 4.300 & 33.30 \\
\hline & & & & 2.850 & 23.20 \\
\hline & & & & 0.950 & 8.25 \\
\hline \multicolumn{2}{|c|}{ k = 12.7} & \multicolumn{2}{|c|}{ k =12.7 } & \multicolumn{2}{|c|}{$\mathbf{k}=7.0$} \\
\hline \multicolumn{2}{|c|}{$\begin{aligned} \Delta \mathbf{k}^{a} & =1.8 \\
\mathbf{R}^{2} & =0.9790\end{aligned}$} & \multicolumn{2}{|c|}{$\begin{aligned} \Delta \mathbf{k}^{a} & =1.2 \\
\mathbf{R}^{2} & =0.9930\end{aligned}$} & \multicolumn{2}{|c|}{$\begin{aligned} \Delta \mathbf{k}^{a} & =0.5 \\
\mathbf{R}^{2} & =0.9913\end{aligned}$} \\
\hline
\end{tabular}

${ }^{a}$ Caculated as the confidence interval of the slope for the $90 \%$ confidence level.

$k^{\text {pivalonitrile }}=13 \pm 1 \quad \mathrm{M}^{-1} \mathrm{~s}^{-1}$

$k^{P h 2 C O / P h H}=7.0 \pm 0.5 \quad M^{-1} s^{-1}$ 
Table S10. Kinetic data for the reaction of $\mathbf{d p p h}^{\bullet}$ with BIS in ethyl acetate. Symbols are the same as described in Table S1.

\begin{tabular}{|c|c|c|c|c|c|}
\hline \multicolumn{2}{|c|}{ ethyl acetate } & \multicolumn{2}{|c|}{ ethyl acetate } & \multicolumn{2}{|c|}{ ethyl acetate } \\
\hline $\begin{array}{l}\text { [BIS] } \\
\text { /mM }\end{array}$ & $\begin{array}{c}\mathrm{k}_{\mathrm{ex}} \times 10^{3} \\
/ \mathrm{s}^{-1}\end{array}$ & $\begin{array}{l}\text { [BIS] } \\
\text { /mM }\end{array}$ & $\begin{array}{c}\mathrm{k}_{\mathrm{ex}} \times 10^{3} \\
/ \mathrm{s}^{-1}\end{array}$ & $\begin{array}{l}{[\mathrm{BIS}]} \\
/ \mathrm{mM}\end{array}$ & $\begin{array}{c}\mathrm{k}_{\mathrm{ex}} \times 10^{3} \\
/ \mathrm{s}^{-1}\end{array}$ \\
\hline 0.925 & 11.330 & 0.925 & 10.840 & 4.453 & 50.165 \\
\hline 0.720 & 9.560 & 0.720 & 8.455 & 2.969 & 29.015 \\
\hline 0.560 & 6.725 & 0.560 & 6.825 & 1.979 & 19.840 \\
\hline 0.435 & 5.415 & 0.435 & 5.555 & 0.880 & 11.795 \\
\hline 0.339 & 3.610 & 0.339 & 4.350 & 0.391 & 6.310 \\
\hline 0.263 & 3.085 & 0.263 & 3.065 & 0.261 & 4.155 \\
\hline 0.205 & 2.450 & 0.205 & 2.290 & 0.174 & 3.415 \\
\hline 0.159 & 1.250 & 0.159 & 1.590 & 0.116 & 2.065 \\
\hline 0.124 & 0.772 & 0.124 & 1.430 & 0.077 & 1.580 \\
\hline 0.096 & 0.753 & 0.096 & 1.030 & & \\
\hline 0.075 & 0.529 & & & & \\
\hline $\mathbf{k}=1$ & & $\mathbf{k}=1$ & & $k=$ & \\
\hline$\Delta \mathbf{k}^{a}=0$ & & $\Delta \mathbf{k}^{a}=0$ & & $\Delta \mathbf{k}^{a}=$ & \\
\hline $\mathbf{R}^{2}=$ & 931 & $\mathbf{R}^{2}=0$ & 956 & $\mathbf{R}^{2}=$ & 9888 \\
\hline
\end{tabular}

${ }^{a}$ Caculated as the confidence interval of the slope for the $90 \%$ confidence level.

$$
k^{\text {ethyl acetate }}=12 \pm 2 \quad \mathrm{M}^{-1} \mathrm{~S}^{-1}
$$

Table S11. Kinetic data for the reaction of $\mathbf{d p p h}^{\bullet}$ with BIS in acidified ethyl acetate. Symbols are the same as described in Table S1.

\begin{tabular}{|c|c|c|c|c|c|}
\hline \multicolumn{2}{|c|}{$\begin{array}{l}\text { ethyl acetate / } \\
10 \text { mM acetic }\end{array}$} & \multicolumn{2}{|c|}{$\begin{array}{l}\text { ethyl acetate / } \\
100 \mathrm{mM} \text { acetic }\end{array}$} & \multicolumn{2}{|c|}{$\begin{array}{c}\text { ethyl acetate / } \\
1000 \text { mM acetic }\end{array}$} \\
\hline $\begin{array}{l}\text { [BIS] } \\
\text { /mM }\end{array}$ & $\begin{array}{c}\mathrm{k}_{\mathrm{ex}} \times 10^{3} \\
/ \mathrm{s}^{-1}\end{array}$ & $\begin{array}{l}\text { [BIS] } \\
/ \mathrm{mM}\end{array}$ & $\begin{array}{c}\mathrm{k}_{\mathrm{ex}} \times 10^{3} \\
/ \mathrm{s}^{-1}\end{array}$ & $\begin{array}{l}\mathrm{BIS}] \\
/ \mathrm{mM} \\
\end{array}$ & $\begin{array}{c}\mathrm{k}_{\mathrm{ex}} \times 10^{3} \\
/ \mathrm{s}^{-1} \\
\end{array}$ \\
\hline 1.25 & 14.0 & 2.65 & 4.16 & 2.65 & 2.47 \\
\hline 0.969 & 9.45 & 2.06 & 3.80 & 2.06 & 2.00 \\
\hline 0.754 & 7.08 & 1.60 & 2.78 & 1.60 & 1.54 \\
\hline 0.586 & 4.64 & 1.25 & 2.43 & 1.25 & 1.20 \\
\hline 0.456 & 2.89 & 0.969 & 1.79 & 0.969 & 0.98 \\
\hline 0.355 & 2.35 & 0.754 & 1.34 & 0.754 & 0.85 \\
\hline 0.276 & 1.82 & 0.586 & 1.01 & 0.586 & 0.67 \\
\hline 0.215 & 1.23 & 0.456 & 0.95 & 0.456 & 0.57 \\
\hline \multirow[t]{2}{*}{0.167} & 1.13 & 0.355 & 0.73 & 0.355 & 0.50 \\
\hline & & 0.276 & 0.74 & 0.276 & 0.36 \\
\hline \multicolumn{2}{|c|}{$\mathbf{k}=11.9$} & \multicolumn{2}{|c|}{$k=1.59$} & \multicolumn{2}{|c|}{$k=0.88$} \\
\hline \multicolumn{2}{|c|}{$\Delta \mathbf{k}^{a}=1.15$} & \multicolumn{2}{|c|}{$\Delta \mathbf{k}^{a}=0.14$} & \multicolumn{2}{|c|}{$\Delta \mathbf{k}^{a}=0.03$} \\
\hline \multicolumn{2}{|c|}{$\mathbf{R}^{2}=0.9808$} & \multicolumn{2}{|c|}{$\mathbf{R}^{2}=0.9826$} & \multicolumn{2}{|c|}{$\mathbf{R}^{2}=0.9979$} \\
\hline
\end{tabular}

${ }^{a}$ Caculated as the confidence interval of the slope for the $90 \%$ confidence level.

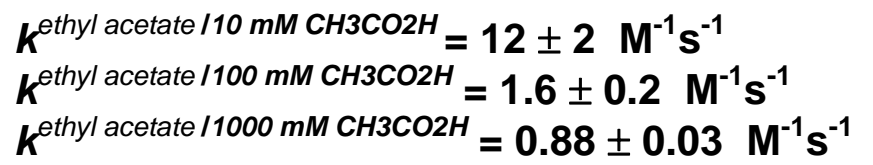


Table S12. Kinetic data for the reaction of dpph $^{\bullet}$ with BIS in dioxane, 3-pentanone and acetophenone. Symbols are the same as described in Table S1.

\begin{tabular}{|c|c|c|c|c|c|}
\hline \multicolumn{2}{|c|}{ dioxane } & \multicolumn{2}{|c|}{ 3-pentanone } & \multicolumn{2}{|c|}{ acetophenone } \\
\hline $\begin{array}{l}\text { [BIS] } \\
/ \mathrm{mM}\end{array}$ & $\begin{array}{c}\mathrm{k}_{\mathrm{ex}} \times 10^{3} \\
/ \mathrm{s}^{-1}\end{array}$ & $\begin{array}{l}{[\mathrm{BIS}]} \\
/ \mathrm{mM}\end{array}$ & $\begin{array}{c}\mathrm{k}_{\mathrm{ex}} \times 10^{3} \\
/ \mathrm{s}^{-1}\end{array}$ & $\begin{array}{l}\text { [BIS] } \\
/ \mathrm{mM}\end{array}$ & $\begin{array}{c}\mathrm{k}_{\mathrm{ex}} \\
/ \mathrm{s}^{-1} \\
\end{array}$ \\
\hline 17.063 & 5.305 & 2.22 & 15.15 & 4.82 & 0.406 \\
\hline 13.271 & 4.150 & 1.72 & 12.58 & 4.30 & 0.340 \\
\hline 9.953 & 3.170 & 1.37 & 9.41 & 3.30 & 0.249 \\
\hline 7.110 & 2.290 & 1.30 & 9.77 & 2.50 & 0.169 \\
\hline 4.740 & 1.555 & 0.93 & 6.99 & 1.77 & 0.105 \\
\hline 2.844 & 0.979 & 0.66 & 4.96 & 1.18 & 0.079 \\
\hline 1.422 & 0.532 & 0.44 & 3.34 & 0.71 & 0.051 \\
\hline 0.711 & 0.282 & & & & \\
\hline $\begin{array}{r}0.355 \\
\mathbf{k}=\end{array}$ & 0.160 & $\mathbf{k}=6$ & & $k=$ & \\
\hline $\begin{aligned} \Delta \mathbf{k}^{a} & =( \\
\mathbf{R}^{2} & =(\end{aligned}$ & $\begin{array}{l}01 \\
9998\end{array}$ & $\begin{aligned} \Delta \mathbf{k}^{a} & =( \\
\mathbf{R}^{2} & =(\end{aligned}$ & $\begin{array}{l}47 \\
9944\end{array}$ & $\begin{aligned} \Delta \mathbf{k}^{a} & = \\
\mathbf{R}^{2} & =\end{aligned}$ & 867 \\
\hline
\end{tabular}

${ }^{a}$ Caculated as the confidence interval of the slope for the $90 \%$ confidence level.

$$
\begin{aligned}
& k^{\text {dioxane }}=0.31 \pm 0.01 \quad \mathrm{M}^{-1} \mathrm{~s}^{-1} \\
& k^{3-\text { pentanone }}=6.5 \pm 0.5 \quad \mathrm{M}^{-1} \mathrm{~s}^{-1} \\
& k^{\text {acetophenone }}=87 \pm 9 \quad \mathrm{M}^{-1} \mathrm{~s}^{-1}
\end{aligned}
$$

Table S13. Kinetic data for the reaction of dpph ${ }^{\bullet}$ with BIS in $\gamma$-valerolactone, pinacolone and THF. Symbols are the same as described in Table S1.

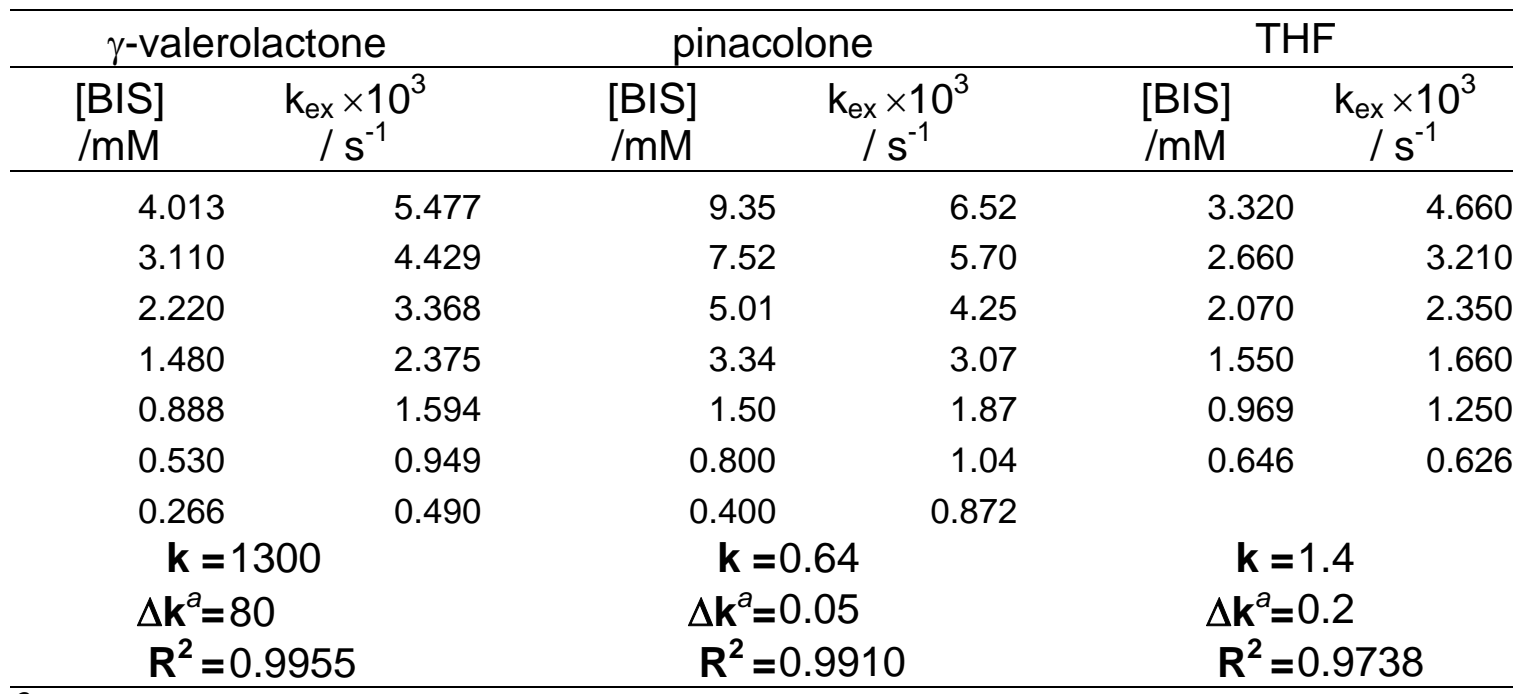

${ }^{a}$ Caculated as the confidence interval of the slope for the $90 \%$ confidence level.

$k^{\gamma \text {-valerolactone }}=1300 \pm 80 \mathrm{M}^{-1} \mathrm{~s}^{-1}$

$k^{\text {pinacolone }}=0.64 \pm 0.1 \mathrm{M}^{-1} \mathrm{~s}^{-1}$

$k^{T H F}=1.4 \pm 0.2 \mathrm{M}^{-1} \mathrm{~s}^{-1}$ 
Table S14. Kinetic data for the reaction of dpph with BIS in acetone and acidified acetone. Symbols are the same as described in Table S1.

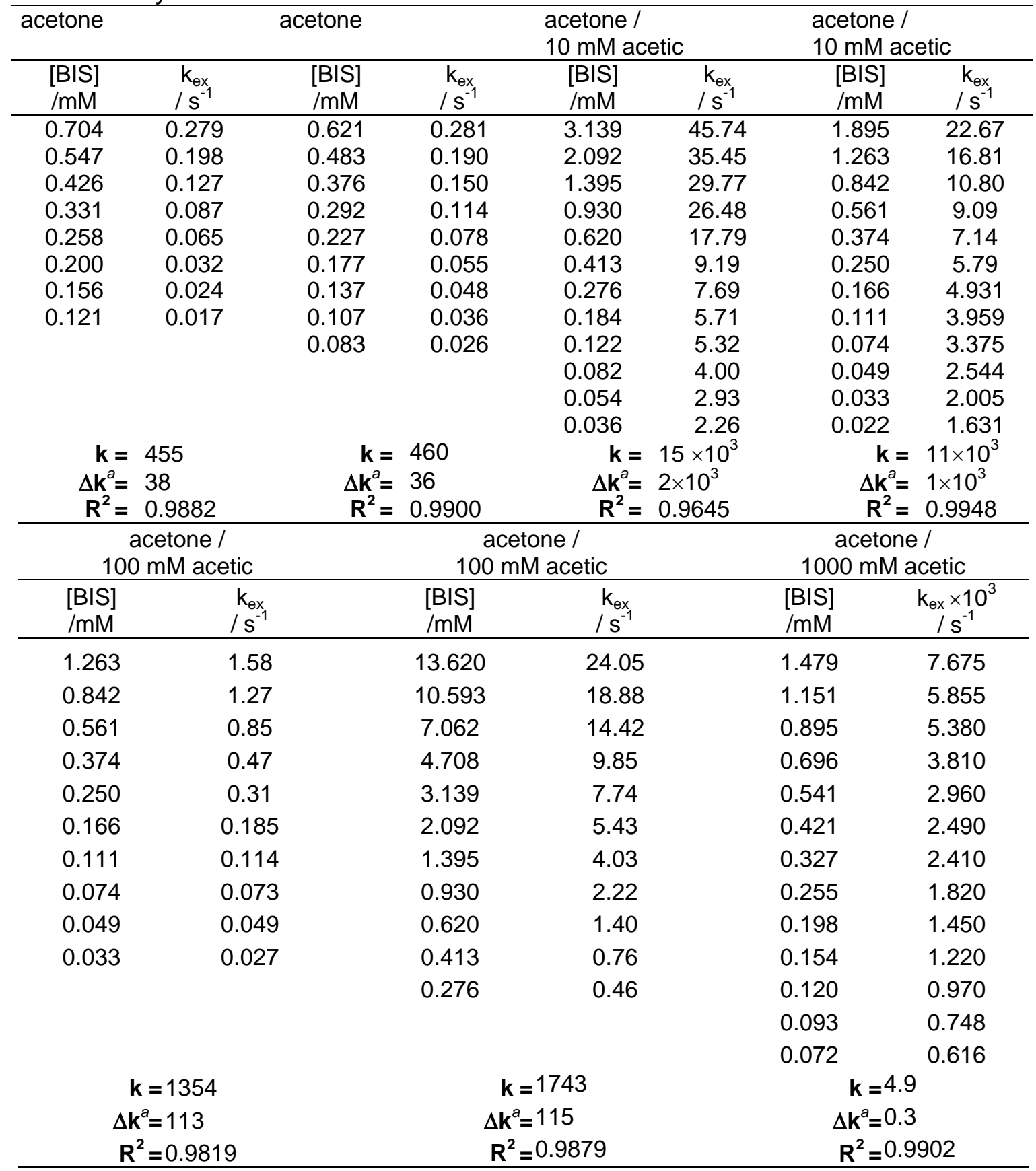

${ }^{a}$ Caculated as the confidence interval of the slope for the $90 \%$ confidence level.

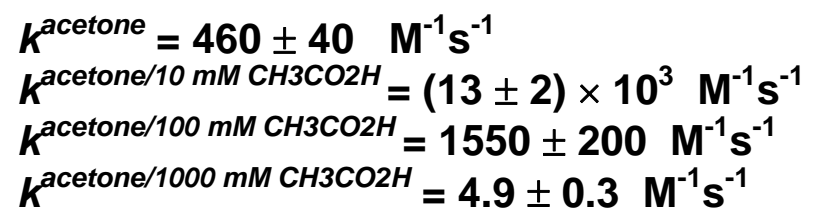


Table S15. Kinetic data for the reaction of $\mathrm{dpph}^{\bullet}$ with BIS in cyclohexanone and dicyclopropylketone. Symbols are the same as described in Table S1.

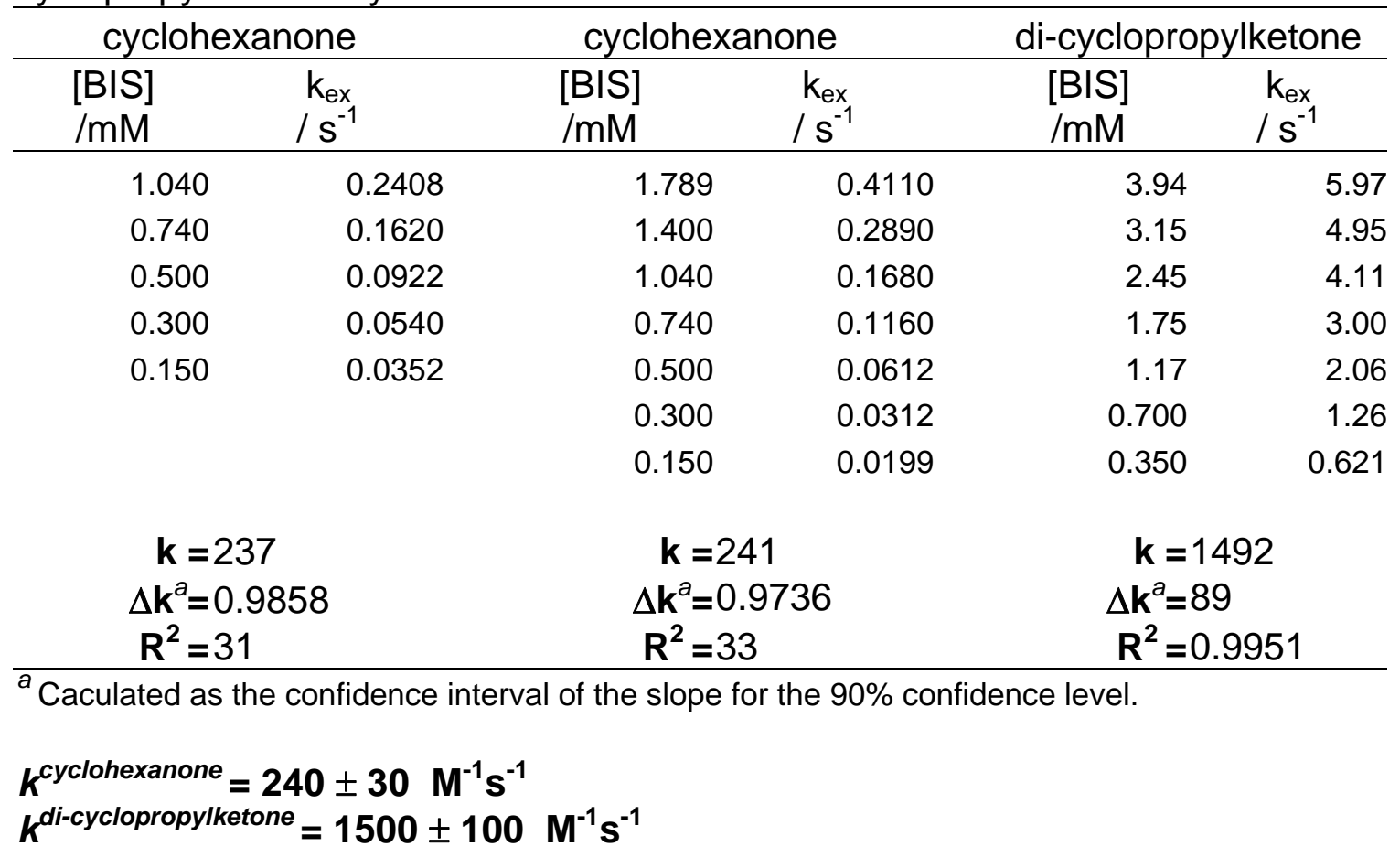

Table S16. Kinetic data for the reaction of $\mathrm{dpph}^{\bullet}$ with BIS in benzonitrile. Symbols are the same as described in Table S1.

\begin{tabular}{|c|c|c|c|}
\hline \multicolumn{2}{|c|}{ benzonitrile } & \multicolumn{2}{|c|}{ benzonitrile } \\
\hline $\begin{array}{l}{[\mathrm{BIS}]} \\
\mathrm{mM}\end{array}$ & $\begin{array}{l}\mathrm{k}_{\mathrm{ex}} \\
/ \mathrm{s}^{-1}\end{array}$ & $\begin{array}{l}{[\mathrm{BIS}]} \\
\mathrm{mM}\end{array}$ & $\begin{array}{l}\mathrm{k}_{\mathrm{ex}} \\
/ \mathrm{s}^{-1}\end{array}$ \\
\hline 1.247 & 0.653 & 3.41 & 2.37 \\
\hline 0.970 & 0.515 & 2.56 & 1.48 \\
\hline 0.754 & 0.416 & 1.83 & 1.02 \\
\hline 0.587 & 0.294 & 1.22 & 0.670 \\
\hline 0.456 & 0.189 & 0.73 & 0.371 \\
\hline 0.215 & 0.109 & 0.44 & 0.244 \\
\hline 0.167 & 0.062 & 0.26 & 0.141 \\
\hline 0.130 & 0.048 & & \\
\hline 0.101 & 0.032 & & \\
\hline 0.079 & 0.023 & & \\
\hline & & & \\
\hline & & & \\
\hline
\end{tabular}

${ }^{a}$ Caculated as the confidence interval of the slope for the $90 \%$ confidence level.

$k^{\text {benzonitrile }}=620 \pm 70 \mathrm{M}^{-1} \mathrm{~s}^{-1}$ 
Table S17. Kinetic data for the reaction of dpph $^{\bullet}$ with BIS in acidified benzonitrile. Symbols are the same as described in Table S1.

\begin{tabular}{|c|c|c|c|}
\hline \multicolumn{2}{|c|}{$\begin{array}{c}\text { Benzonitrile } \\
\text { 10mM acetic acid }\end{array}$} & \multicolumn{2}{|c|}{$\begin{array}{c}\text { Benzonitrile } \\
\text { 10mM acetic acid }\end{array}$} \\
\hline $\begin{array}{l}\text { [BIS] } \\
/ \mathrm{mM}\end{array}$ & $\begin{array}{l}k_{\mathrm{ex}} \\
/ \mathrm{s}^{-1}\end{array}$ & $\begin{array}{l}\text { [BIS] } \\
/ \mathrm{mM}\end{array}$ & $\begin{array}{l}\mathrm{k}_{\mathrm{ex}} \\
/ \mathrm{s}^{-1}\end{array}$ \\
\hline 1.85 & 1.350 & 1.04 & 0.773 \\
\hline 1.44 & 1.093 & 0.808 & 0.544 \\
\hline 1.12 & 0.823 & 0.628 & 0.461 \\
\hline 0.871 & 0.644 & 0.489 & 0.362 \\
\hline 0.678 & 0.502 & 0.380 & 0.232 \\
\hline 0.527 & 0.379 & 0.296 & 0.181 \\
\hline 0.376 & 0.271 & 0.230 & 0.116 \\
\hline 0.251 & 0.176 & 0.179 & 0.081 \\
\hline 0.151 & 0.110 & 0.139 & 0.060 \\
\hline \multirow[t]{4}{*}{0.075} & 0.056 & 0.108 & 0.052 \\
\hline & & 0.084 & 0.049 \\
\hline & & 0.054 & 0.029 \\
\hline & & 0.042 & 0.022 \\
\hline & & \multicolumn{2}{|c|}{$\begin{aligned} \Delta \mathbf{k}^{a} & =38 \\
\mathbf{R}^{2} & =0.9916\end{aligned}$} \\
\hline
\end{tabular}

\begin{tabular}{|c|c|c|c|c|c|}
\hline \multicolumn{2}{|c|}{$\begin{array}{c}\text { Benzonitrile } \\
100 \mathrm{mM} \text { acetic acid }\end{array}$} & \multicolumn{2}{|c|}{$\begin{array}{c}\text { Benzonitrile } \\
100 \mathrm{mM} \text { acetic acid }\end{array}$} & \multicolumn{2}{|c|}{$\begin{array}{c}\text { Benzonitrile } \\
1000 \text { mM acetic acid }\end{array}$} \\
\hline $\begin{array}{l}{[\mathrm{BIS}]} \\
/ \mathrm{mM}\end{array}$ & $\begin{array}{l}k_{\mathrm{ex}} \\
/ \mathrm{s}^{-1}\end{array}$ & $\begin{array}{l}{[\mathrm{BIS}]} \\
/ \mathrm{mM}\end{array}$ & $\begin{array}{l}\mathrm{k}_{\mathrm{ex}} \\
/ \mathrm{s}^{-1}\end{array}$ & $\begin{array}{l}\text { [BIS] } \\
\text { /mM }\end{array}$ & $\begin{array}{l}k_{e x} \\
/ \mathrm{s}^{-1}\end{array}$ \\
\hline 0.507 & 16.1 & 1.157 & 27.81 & 0.720 & 18.265 \\
\hline 0.394 & 11.3 & 0.900 & 23.40 & 0.560 & 17.610 \\
\hline 0.307 & 10.8 & 0.700 & 18.68 & 0.436 & 16.170 \\
\hline 0.239 & 9.74 & 0.544 & 15.01 & 0.339 & 14.700 \\
\hline 0.186 & 9.03 & 0.423 & 11.62 & 0.263 & 12.940 \\
\hline 0.112 & 7.21 & 0.329 & 9.350 & 0.188 & 11.770 \\
\hline \multirow[t]{2}{*}{0.056} & 4.74 & 0.235 & 7.020 & 0.125 & 10.510 \\
\hline & & 0.157 & 5.020 & 0.075 & 8.720 \\
\hline \multicolumn{2}{|c|}{$\begin{aligned} \mathbf{k} & =22 \\
\Delta \mathbf{k}^{a} & =5 \\
\mathbf{R}^{2} & =0.9397\end{aligned}$} & \multicolumn{2}{|c|}{$\begin{aligned} \mathbf{k} & =24 \\
\Delta \mathbf{k}^{a} & =1 \\
\mathbf{R}^{2} & =0.9956\end{aligned}$} & \multicolumn{2}{|c|}{$\begin{aligned} \mathbf{k} & =15 \\
\Delta \mathbf{k}^{a} & =3 \\
\mathbf{R}^{2} & =0.9410\end{aligned}$} \\
\hline
\end{tabular}

${ }^{a}$ Caculated as the confidence interval of the slope for the $90 \%$ confidence level.

$$
\begin{aligned}
& k^{\text {benzonitrile } / 10 \mathrm{mM} \mathrm{CH} C \mathrm{CO} H}=750 \pm 30 \mathrm{M}^{-1} \mathrm{~s}^{-1} \\
& k^{\text {benzonitrile/ } 100 \mathrm{mM} \mathrm{CH} 3 \mathrm{CO} H}=23 \pm 4 \mathrm{M}^{-1} \mathrm{~s}^{-1} \\
& k^{\text {benzonitrile } / 1000 \mathrm{mM} \mathrm{CH} 3 \mathrm{CO} 2 \mathrm{H}}=15 \pm 3 \mathrm{M}^{-1} \mathrm{~s}^{-1}
\end{aligned}
$$


Table S18. Kinetic data for the reaction of dpph ${ }^{\bullet}$ with BIS in DMSO. Symbols are the same as described in Table S1.

\begin{tabular}{|c|c|c|c|c|c|}
\hline \multicolumn{2}{|c|}{ DMSO } & \multicolumn{2}{|c|}{ DMSO } & \multicolumn{2}{|c|}{ DMSO } \\
\hline $\begin{array}{l}\text { [BIS] } \\
/ \mathrm{mM}\end{array}$ & $\begin{array}{l}\mathrm{k}_{\mathrm{ex}} \\
/ \mathrm{s}^{-1}\end{array}$ & $\begin{array}{l}\text { [BIS] } \\
/ \mathrm{mM}\end{array}$ & $\begin{array}{c}\mathrm{k}_{\mathrm{ex}} \\
/ \mathrm{s}^{-1} \\
\end{array}$ & $\begin{array}{l}\text { [BIS] } \\
/ \mathrm{mM} \\
\end{array}$ & $\begin{array}{l}\mathrm{k}_{\mathrm{ex}} \\
/ \mathrm{s}^{-1} \\
\end{array}$ \\
\hline 3.94 & 0.763 & 4.49 & 0.687 & 4.49 & 0.692 \\
\hline 3.06 & 0.602 & 3.49 & 0.502 & 3.49 & 0.509 \\
\hline 2.38 & 0.521 & 2.72 & 0.442 & 2.72 & 0.411 \\
\hline 1.85 & 0.404 & 2.11 & 0.343 & 2.11 & 0.302 \\
\hline 1.44 & 0.304 & 1.64 & 0.287 & 1.64 & 0.259 \\
\hline 1.12 & 0.268 & 1.28 & 0.233 & 1.28 & 0.211 \\
\hline 0.872 & 0.193 & 0.994 & 0.201 & 0.994 & 0.148 \\
\hline 0.678 & 0.149 & 0.773 & 0.159 & 0.773 & 0.127 \\
\hline 0.527 & 0.108 & 0.601 & 0.131 & 0.601 & 0.110 \\
\hline \multirow[t]{2}{*}{0.410} & 0.097 & 0.468 & 0.110 & 0.468 & 0.093 \\
\hline & & 0.364 & 0.096 & 0.364 & 0.061 \\
\hline \multicolumn{2}{|c|}{$\mathbf{k}=193$} & \multicolumn{2}{|c|}{$k=139$} & \multicolumn{2}{|c|}{$k=147$} \\
\hline \multicolumn{2}{|c|}{$\begin{aligned} \Delta \mathbf{k}^{a} & =10 \\
\mathbf{R}^{2} & =0.9929\end{aligned}$} & \multicolumn{2}{|c|}{$\begin{aligned} \Delta \mathbf{k}^{a} & =5 \\
\mathbf{R}^{2} & =0.9954\end{aligned}$} & \multicolumn{2}{|c|}{$\begin{aligned} \Delta \mathbf{k}^{a} & =5 \\
\mathbf{R}^{2} & =0.9963\end{aligned}$} \\
\hline
\end{tabular}

${ }^{a}$ Caculated as the confidence interval of the slope for the $90 \%$ confidence level.

$k^{D M S O}=160 \pm 30 M^{-1} s^{-1}$

Table S19. Kinetic data for the reaction of $\mathbf{d p p h}^{\bullet}$ with BIS in acidified DMSO. Symbols are the same as described in Table S1.

\begin{tabular}{|c|c|c|c|c|c|c|c|}
\hline \multicolumn{2}{|c|}{$\begin{array}{l}\text { DMSO / } \\
10 \text { mM acetic }\end{array}$} & \multicolumn{2}{|c|}{$\begin{array}{l}\text { DMSO / } \\
10 \text { mM acetic }\end{array}$} & \multicolumn{2}{|c|}{$\begin{array}{l}\text { DMSO / } \\
100 \mathrm{mM} \text { acetic }\end{array}$} & \multicolumn{2}{|c|}{$\begin{array}{l}\text { DMSO / } \\
100 \text { mM acetic }\end{array}$} \\
\hline $\begin{array}{l}{[\mathrm{BIS}]} \\
/ \mathrm{mM}\end{array}$ & $\begin{array}{l}\mathrm{k}_{\mathrm{ex}} \\
/ \mathrm{s}^{-1}\end{array}$ & $\begin{array}{l}\text { [BIS] } \\
/ \mathrm{mM}\end{array}$ & $\begin{array}{l}\mathrm{k}_{\mathrm{ex}} \\
/ \mathrm{s}^{-1}\end{array}$ & $\begin{array}{l}{[\mathrm{BIS}]} \\
/ \mathrm{mM}\end{array}$ & $\begin{array}{l}k_{\mathrm{ex}} \\
/ \mathrm{s}^{-1}\end{array}$ & $\begin{array}{l}\text { [BIS] } \\
/ \mathrm{mM}\end{array}$ & $\begin{array}{l}\mathrm{k}_{\mathrm{ex}} \\
/ \mathrm{s}^{-1}\end{array}$ \\
\hline 1.552 & 2.007 & 1.554 & 2.457 & 1.207 & 5.605 & 1.554 & 8.223 \\
\hline 1.207 & 1.582 & 1.208 & 2.125 & 0.939 & 5.047 & 1.208 & 6.947 \\
\hline 0.939 & 1.308 & 0.906 & 2.008 & 0.730 & 4.043 & 0.906 & 5.770 \\
\hline 0.730 & 1.219 & 0.647 & 1.756 & 0.568 & 3.544 & 0.647 & 4.400 \\
\hline 0.568 & 1.099 & 0.432 & 1.511 & 0.442 & 2.773 & 0.432 & 2.742 \\
\hline 0.442 & 0.891 & 0.288 & 1.356 & 0.344 & 2.369 & 0.288 & 1.885 \\
\hline 0.344 & 0.883 & 0.173 & 1.272 & 0.267 & 1.631 & 0.173 & 1.084 \\
\hline 0.267 & 0.767 & & & 0.208 & 1.480 & & \\
\hline 0.208 & 0.764 & & & 0.162 & 1.311 & & \\
\hline 0.162 & 0.605 & & & 0.126 & 0.872 & & \\
\hline $\mathrm{k}=$ & 20 & $\mathbf{k}=$ & 854 & $\mathbf{k}=$ & 500 & $k=$ & 5200 \\
\hline $\begin{aligned} \Delta \mathbf{k}^{\mathrm{a}}= \\
\mathbf{R}^{2}=\end{aligned}$ & 22 & $\begin{aligned} \Delta \mathbf{k}^{a} & = \\
\mathbf{R}^{2} & =\end{aligned}$ & & $\begin{aligned} \Delta \mathbf{k}^{a} & = \\
R^{2} & =\end{aligned}$ & 60 & $\begin{aligned} \Delta \mathbf{k}^{a} & = \\
\mathbf{R}^{2} & =\end{aligned}$ & 620 \\
\hline & & & & & & & \\
\hline
\end{tabular}

\footnotetext{
${ }^{a}$ Caculated as the confidence interval of the slope for the $90 \%$ confidence level.
} 
Table S19.continued

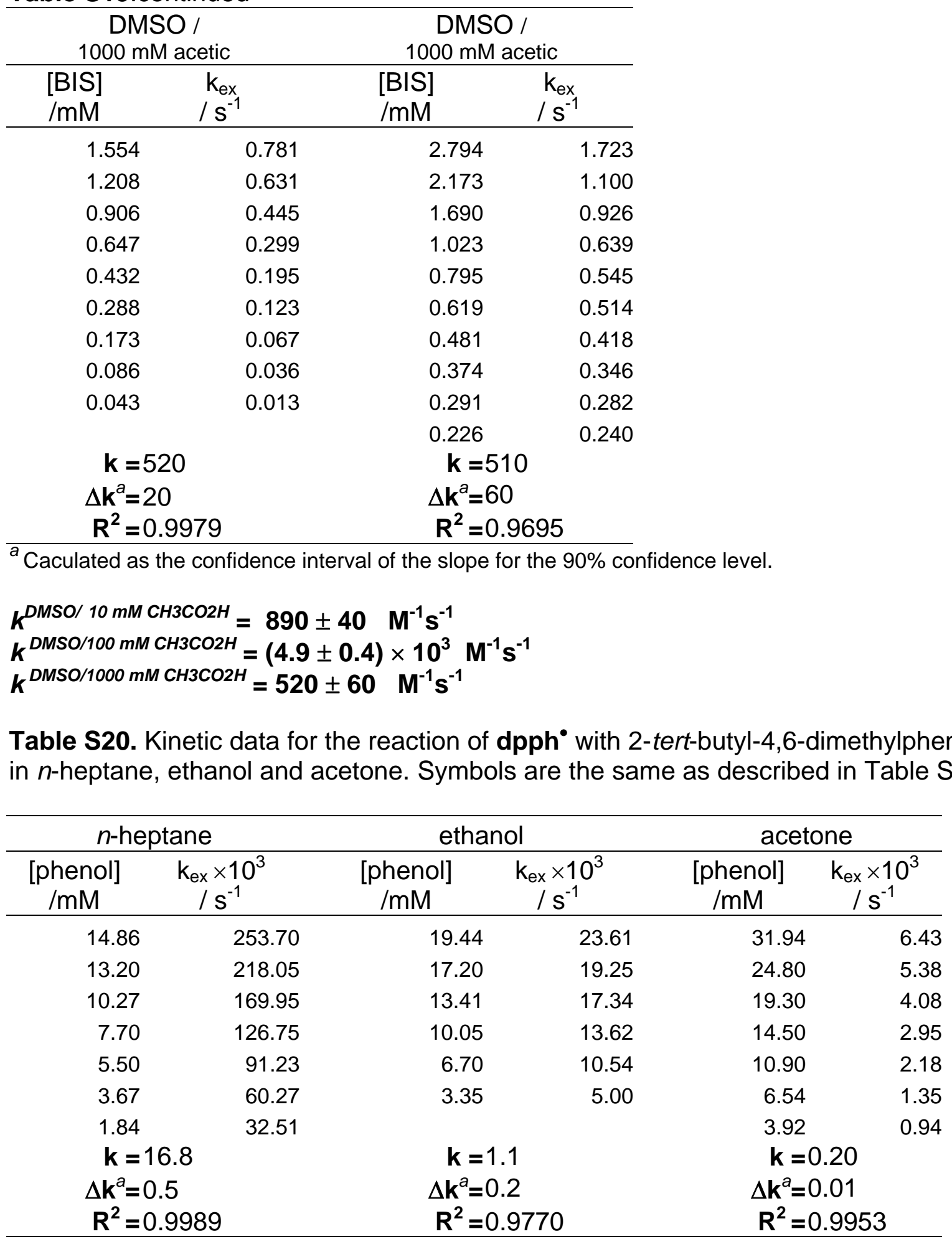

${ }^{a}$ Caculated as the confidence interval of the slope for the $90 \%$ confidence level.

$$
\begin{aligned}
& k^{n-h e p t a n e}=17 \pm 1 \mathrm{M}^{-1} \mathrm{~s}^{-1} \\
& k^{\text {ethanol }}=1.1 \pm 0.2 \mathrm{M}^{-1} \mathrm{~s}^{-1} \\
& k^{\text {acetone }}=0.20 \pm 0.01 \mathrm{M}^{-1} \mathrm{~s}^{-1}
\end{aligned}
$$



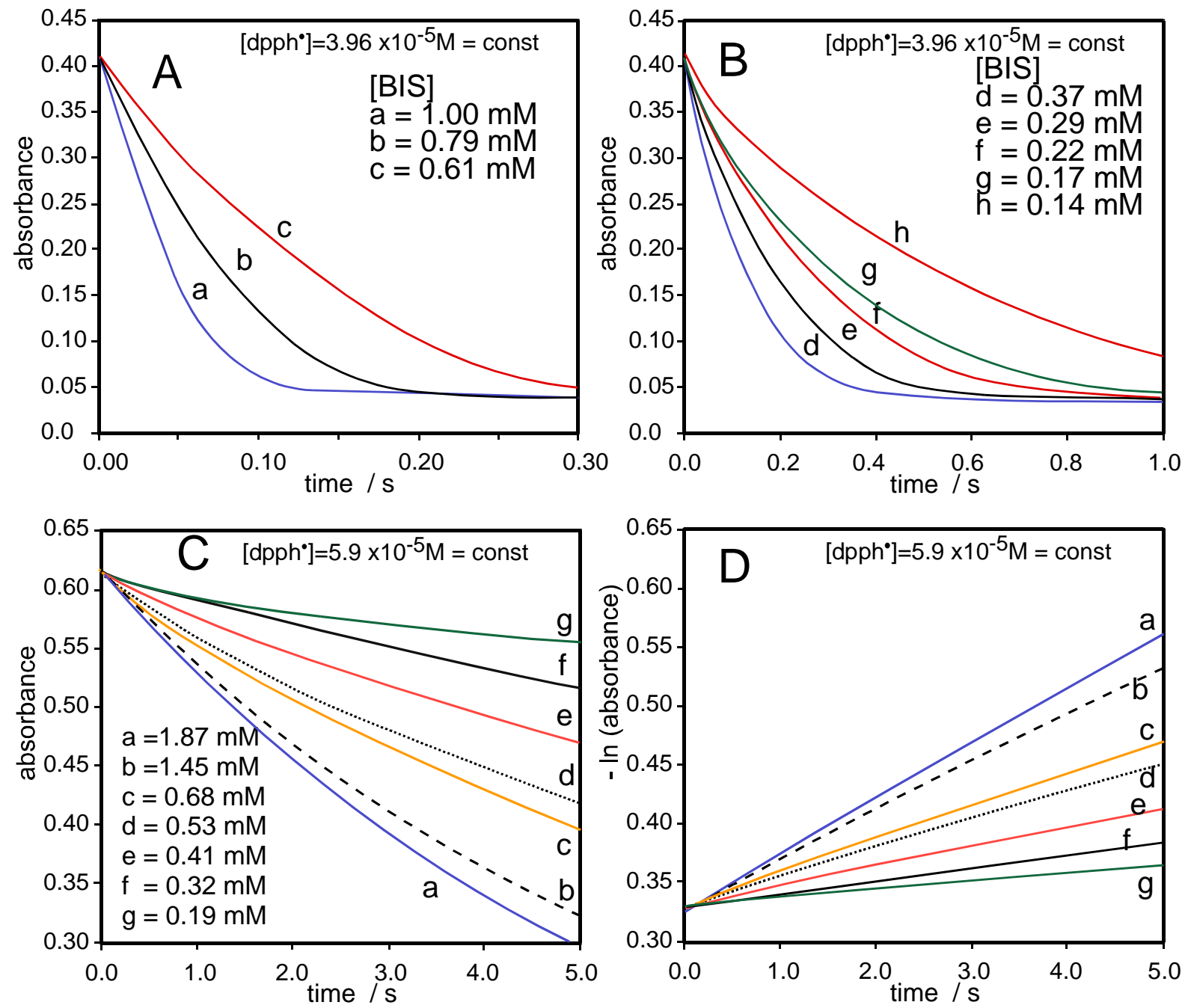

Figure S1.

Decay of $\mathbf{d p p h}^{\bullet}$ in reaction with BIS in neat acetonitrile (panels A and B) with different concentration of BIS. Panels $C$ and D present the plots of absorbance (Panel $\mathrm{C}$ ) and negative logarithm from absorbance for reactions in acidified acetonitrile $\left(\left[\mathrm{CH}_{3} \mathrm{CO}_{2} \mathrm{H}\right]=100 \mathrm{mM}\right)$, Panel D. 
Table S21. Kinetic data for the reaction of dpph ${ }^{\bullet}$ with 2,4,6-trimethylphenol in several solvents. Symbols are the same as described in Table S1.

\begin{tabular}{lccc}
\hline \multicolumn{1}{c}{ solvent } & $\beta_{2}^{H}$ & $\boldsymbol{k}^{\boldsymbol{S}}$ & $\log \boldsymbol{k}^{\boldsymbol{S}}$ \\
\hline heptane & & & \\
CCl4 & 0.00 & 40 & 1.60 \\
methyl formate & 0.05 & 23 & 1.35 \\
methanol & 0.38 & 0.71 & -0.15 \\
acetonitrile & 0.41 & 43 & 1.63 \\
ethanol & 0.44 & 0.56 & -0.25 \\
pivalonitrile & 0.44 & 13 & 1.11 \\
ethyl acetate & 0.44 & 0.70 & -0.15 \\
dioxane & 0.47 & 0.55 & -0.26 \\
acetone & 0.5 & 0.36 & -0.44 \\
tetrahydrofuran & 0.51 & 0.31 & -0.51 \\
dicyclopropketone & 0.53 & 0.24 & -0.62 \\
$\gamma$-valerolactone & 0.55 & 0.65 & 0.16 \\
DMSO & 0.78 & 0.031 & -0.19 \\
\hline
\end{tabular}

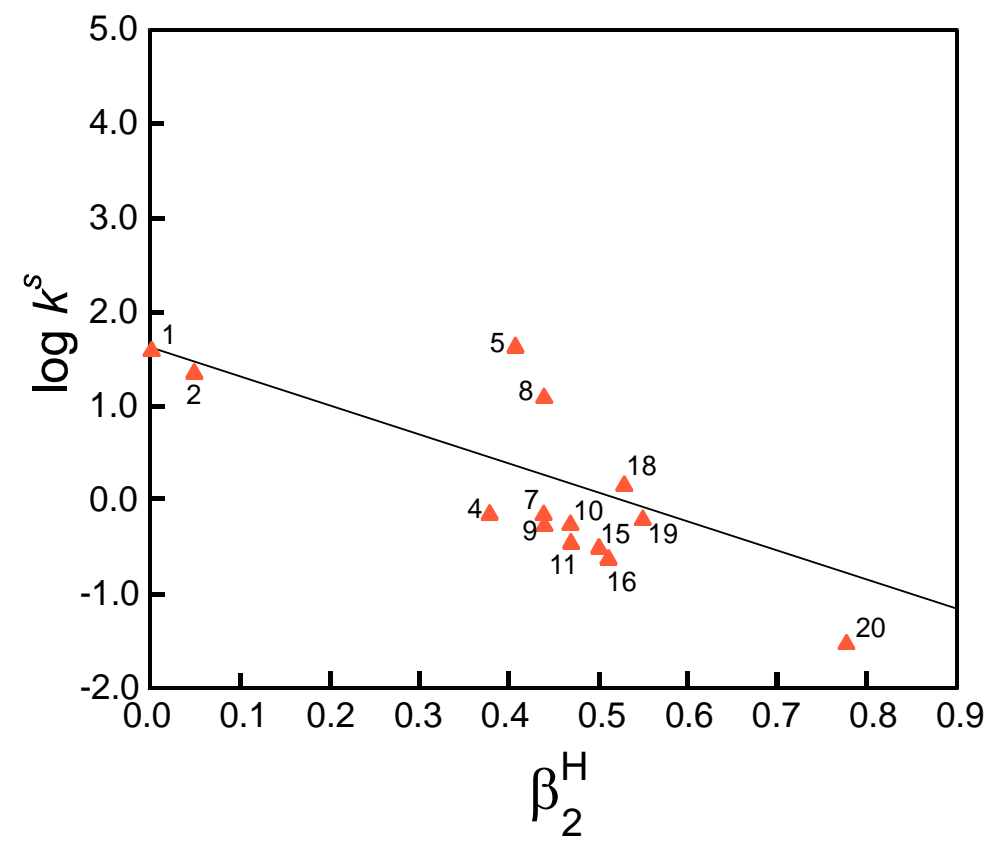

Figure S2. Plots of $\log k^{\mathrm{s}}$ for for the reaction of $\mathbf{d p p h}^{\bullet}$ with 2,4,6-trimethylphenol in the solvents from Table S21 vs. $\beta_{2}^{H}$. The straight line was constructed from equation II, taking $\alpha_{2}^{H}=0.37$ for 2,4,6-Me 3 phenol 
Table S22. Parameters used for calculation of the equilibrium constant $K$ for HB complex formation between DMSO and BIS. $[\mathrm{ArOH}]_{\mathrm{o}}=$ total concentration of phenol, $[\mathrm{ArOH}]_{\text {free }}=$ concentration of non-hydrogen bonded phenol calculated from IR measurements, $[\mathrm{HB}]=[\mathrm{ArOH}]_{0}-[\mathrm{ArOH}]_{\text {free, }},[\mathrm{DMSO}]_{0}=$ total concentration of DMSO in $\mathrm{CCl}_{4},[\mathrm{DMSO}]_{\text {free }}=$ concentration of free (i.e. non-hydrogen bonded) DMSO. Plots of $[\mathrm{ArOH}]_{0} /[\mathrm{ArOH}]_{\text {free }}$ vs. [DMSO $]_{\text {free }}$ are presented in Figure S3. All concentrations are given in $\mathrm{mM}$ units.

\begin{tabular}{|c|c|c|c|c|c|}
\hline$[\mathrm{ArOH}]_{0}$ & {$[\mathrm{ArOH}]_{\text {free }}$} & {$[\mathrm{HB}]$} & {$[\mathrm{DMSO}]_{0}$} & {$[\mathrm{DMSO}]_{\text {free }}$} & {$[\mathrm{ArOH}]_{\mathrm{o}} /[\mathrm{ArOH}]_{\text {free }}$} \\
\hline \multicolumn{6}{|c|}{$\mathrm{BIS}+\mathrm{DMSO}$} \\
\hline 17.41 & 3.0 & 14.4 & 90.6 & 76.16 & 5.85 \\
\hline 17.41 & 3.5 & 14.0 & 73.5 & 59.53 & 5.04 \\
\hline 17.41 & 3.8 & 13.6 & 59.6 & 45.97 & 4.62 \\
\hline 17.41 & 4.8 & 12.6 & 48.3 & 35.73 & 3.63 \\
\hline 17.41 & 6.1 & 11.3 & 39.2 & 27.91 & 2.85 \\
\hline 17.41 & 6.8 & 10.6 & 31.8 & 21.19 & 2.56 \\
\hline 17.41 & 7.2 & 10.3 & 25.8 & 15.55 & 2.43 \\
\hline 17.41 & 8.2 & 9.3 & 20.9 & 11.67 & 2.13 \\
\hline 17.41 & 17.4 & 0.0 & 0.0 & 0.00 & 1.00 \\
\hline \multicolumn{6}{|c|}{ BIS + DMSO } \\
\hline 17.41 & 4.0 & 13.4 & 90.0 & 76.63 & 4.31 \\
\hline 17.41 & 4.7 & 12.7 & 75.0 & 62.26 & 3.73 \\
\hline 17.41 & 5.3 & 12.1 & 62.5 & 50.42 & 3.26 \\
\hline 17.41 & 6.1 & 11.3 & 52.1 & 40.81 & 2.84 \\
\hline 17.41 & 6.9 & 10.5 & 43.4 & 32.90 & 2.52 \\
\hline 17.41 & 8.2 & 9.2 & 34.7 & 25.50 & 2.13 \\
\hline 17.41 & 17.4 & 0.0 & 0.0 & 0.00 & 1.00 \\
\hline \multicolumn{6}{|c|}{ BIS + DMSO } \\
\hline 13.40 & 13.4 & 0.0 & 0.0 & 0 & 1.00 \\
\hline 13.40 & 2.0 & 11.4 & 98.4 & 87.04 & 6.56 \\
\hline 13.40 & 2.7 & 10.7 & 76.5 & 65.84 & 4.95 \\
\hline 13.40 & 3.4 & 10.0 & 57.4 & 47.37 & 3.97 \\
\hline 13.40 & 4.2 & 9.2 & 43.1 & 33.82 & 3.21 \\
\hline 13.40 & 5.2 & 8.2 & 32.3 & 24.04 & 2.60 \\
\hline 13.40 & 6.3 & 7.1 & 24.2 & 17.09 & 2.14 \\
\hline 13.40 & 7.2 & 6.2 & 18.2 & 11.91 & 1.87 \\
\hline 13.40 & 8.2 & 5.2 & 13.6 & 8.47 & 1.62 \\
\hline 13.40 & 9.6 & 3.8 & 9.1 & 5.29 & 1.39 \\
\hline
\end{tabular}



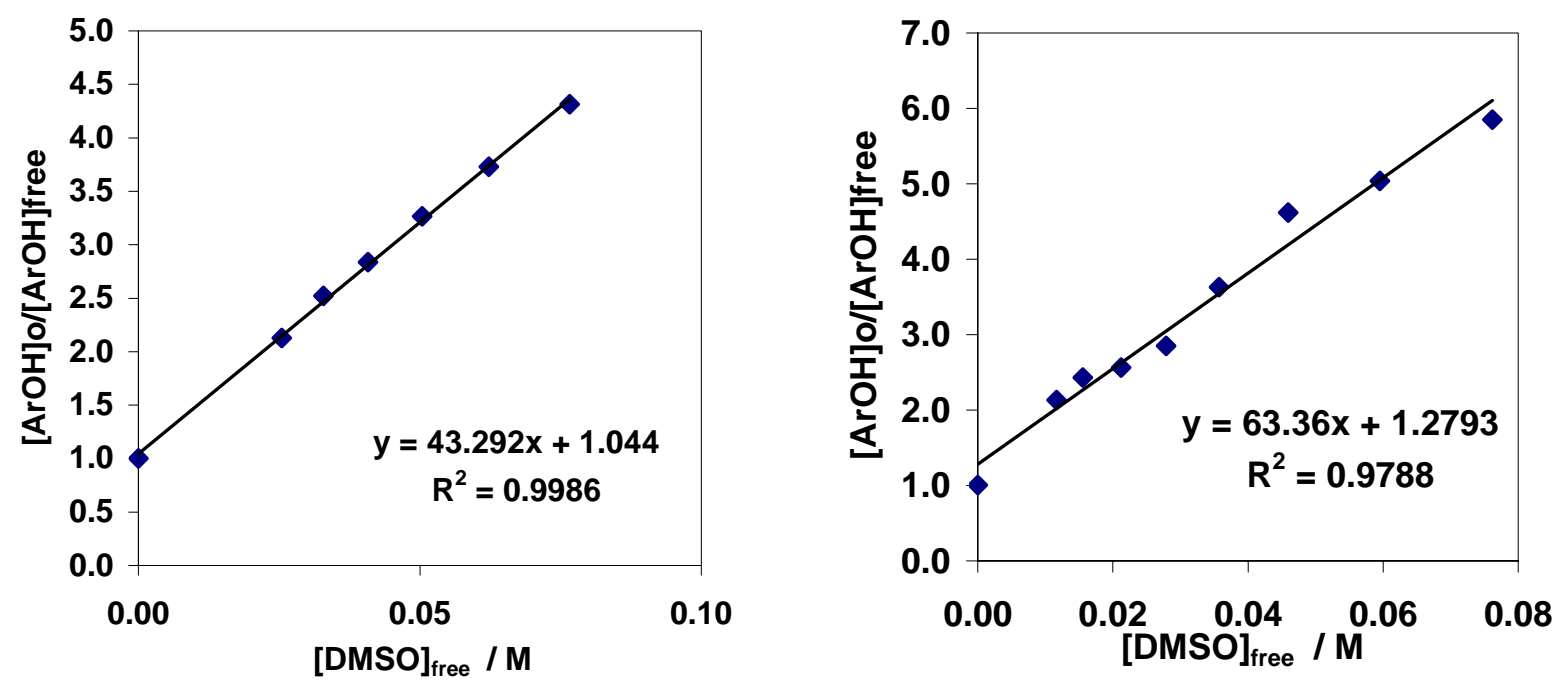

a)

b)

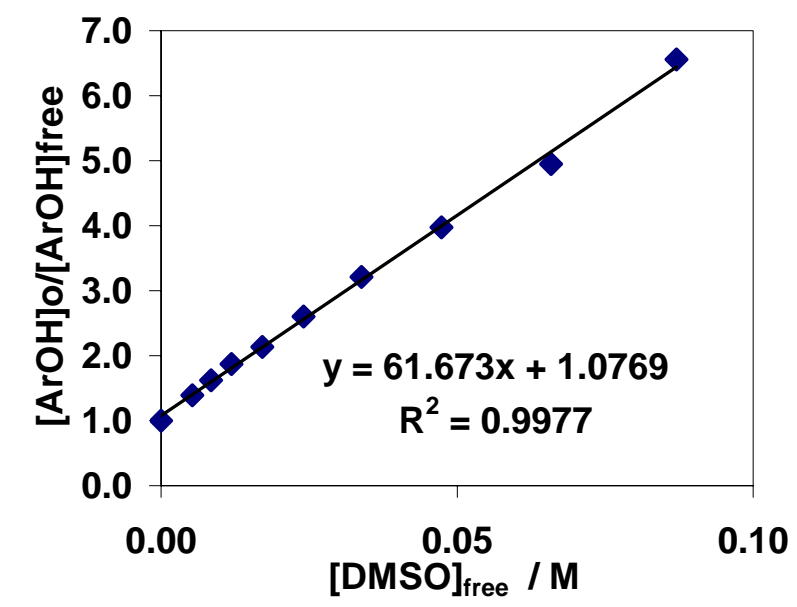

c)

Figure S3. Plots of $[\mathrm{ArOH}]_{0} /[\mathrm{ArOH}]_{\text {tree }}$ vs. [DMSO $]_{\text {ree }}$ for BIS. The slopes of the straight lines yield the HB equilibrium constant, $K$. 
Table S23. Parameters used for calculation of the equilibrium constant $K$ for HB complex formation between BIS and acetonitrile $(\mathrm{ACN}) .[\mathrm{ArOH}]_{0}=$ total concentration of phenol, $[\mathrm{ArOH}]_{\text {free }}=$ concentration of non-hydrogen bonded phenol calculated from IR measurements, $[\mathrm{HB}]=[\mathrm{ArOH}]_{0}-[\mathrm{ArOH}]_{\text {free, }},[\mathrm{ACN}]_{0}$ $=$ total concentration of acetonitrile in $\mathrm{CCl}_{4},[\mathrm{ACN}]_{\text {free }}=$ concentration of free (i.e. non-hydrogen bonded) acetonitrile. Plots of $[\mathrm{ArOH}]_{\mathrm{o}} /[\mathrm{ArOH}]_{\text {free }}$ vs. $[\mathrm{ACN}]_{\text {free }}$ are presented in Figure S4. All concentrations are given in $\mathrm{mM}$ units.

\begin{tabular}{cccccc}
\hline$[\mathrm{ArOH}]_{\mathrm{o}}$ & {$[\mathrm{ArOH}]_{\text {free }}$} & {$[\mathrm{HB}]$} & {$[\mathrm{ACN}]_{\mathrm{o}}$} & {$[\mathrm{ACN}]_{\text {free }}$} & {$[\mathrm{ArOH}]_{\mathrm{o}} /[\mathrm{ArOH}]_{\text {free }}$} \\
\hline \multicolumn{5}{c}{$\mathrm{BIS}+$ acetonitrile } \\
18.40 & 13.0 & 5.4 & 266.0 & 260.63 & 1.41 \\
18.40 & 14.8 & 3.6 & 159.6 & 155.99 & 1.24 \\
18.40 & 15.9 & 2.5 & 95.8 & 93.29 & 1.15 \\
18.40 & 17.0 & 1.4 & 57.5 & 56.10 & 1.08 \\
18.40 & 17.7 & 0.7 & 31.9 & 31.19 & 1.04 \\
18.40 & 18.4 & 0.0 & 0.0 & 0.00 & 1.00 \\
& \multicolumn{5}{c}{} \\
15.80 & 8.0 & 7.8 & 643.7 & 635.84 & \\
15.80 & 8.9 & 6.9 & 482.7 & 475.88 & 1.98 \\
15.80 & 10.4 & 5.4 & 344.8 & 339.37 & 1.77 \\
15.80 & 11.6 & 4.2 & 229.9 & 225.72 & 1.53 \\
15.80 & 12.0 & 3.8 & 153.3 & 149.48 & 1.36 \\
15.80 & 12.9 & 2.9 & 102.2 & 99.30 & 1.31 \\
15.80 & 13.1 & 2.7 & 68.1 & 65.40 & 1.22 \\
15.80 & 14.0 & 1.8 & 45.4 & 43.60 & 1.21 \\
15.80 & 15.8 & 0.0 & 0.0 & 0.00 & 1.13 \\
\hline
\end{tabular}
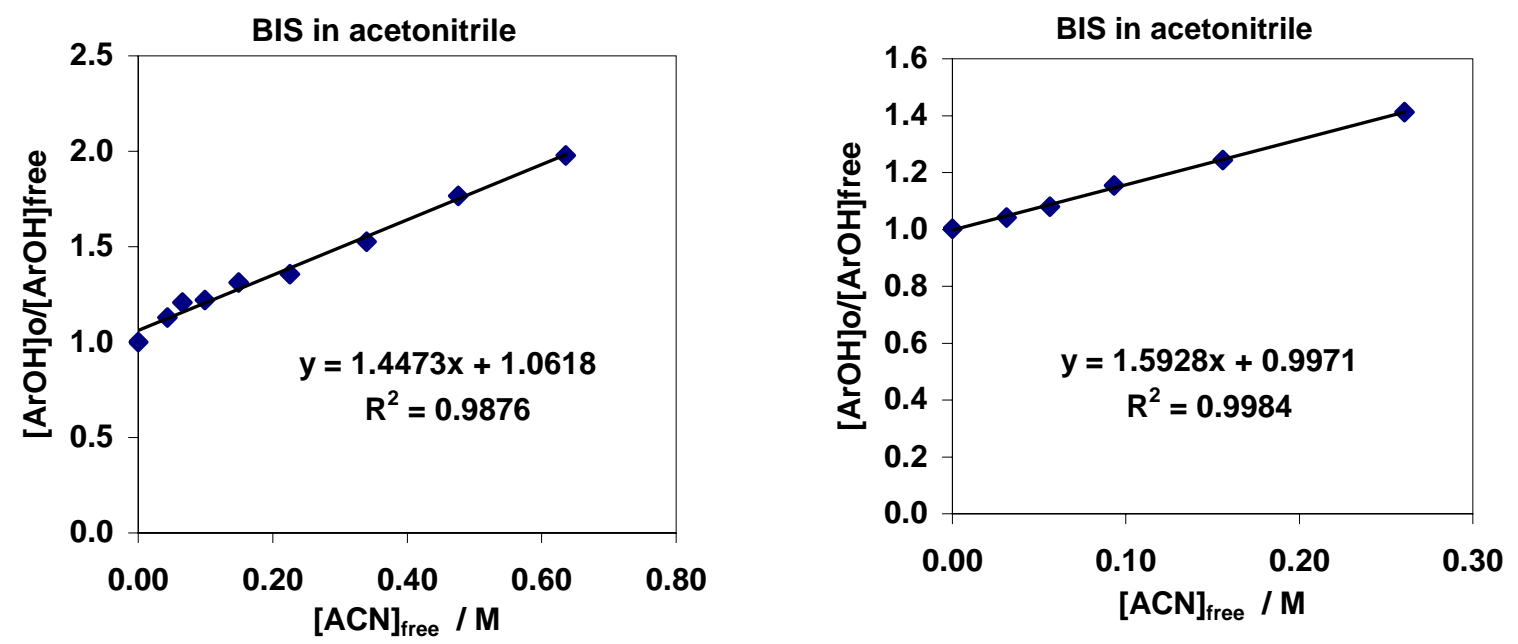

Figure S4. Plots of $[\mathrm{ArOH}]_{\mathrm{o}} /[\mathrm{ArOH}]_{\text {free }}$ vs. $[\mathrm{ACN}]_{\text {free }}$ for BIS. The slopes of the straight lines yield the HB equilibrium constant, $K$. 
Table S24. Values $K, \log K_{A}^{H_{i}}$, and $\alpha_{2}^{H}$ for BIS calculated from IR measurements of HB complex formation with DMSO and acetonitrile.

\begin{tabular}{cccccc}
\hline solvent & $L_{\mathrm{B}}{ }^{a}$ & $D_{\mathrm{B}}{ }^{a}$ & $K$ & $\log K_{A}^{H a}$ & $\alpha_{2}^{H}$ \\
\hline DMSO & 1.24 & 0.266 & 60.56 & 1.223 & 0.501 \\
DMSO & 1.24 & 0.266 & 41.60 & 1.092 & 0.473 \\
DMSO & 1.24 & 0.266 & 61.67 & 1.230 & 0.503 \\
acetonitrile & 0.688 & -0.340 & 1.59 & 0.787 & 0.407 \\
acetonitrile & 0.688 & -0.340 & 1.45 & 0.728 & 0.394 \\
\hline
\end{tabular}

${ }^{a}$ Reference 10.

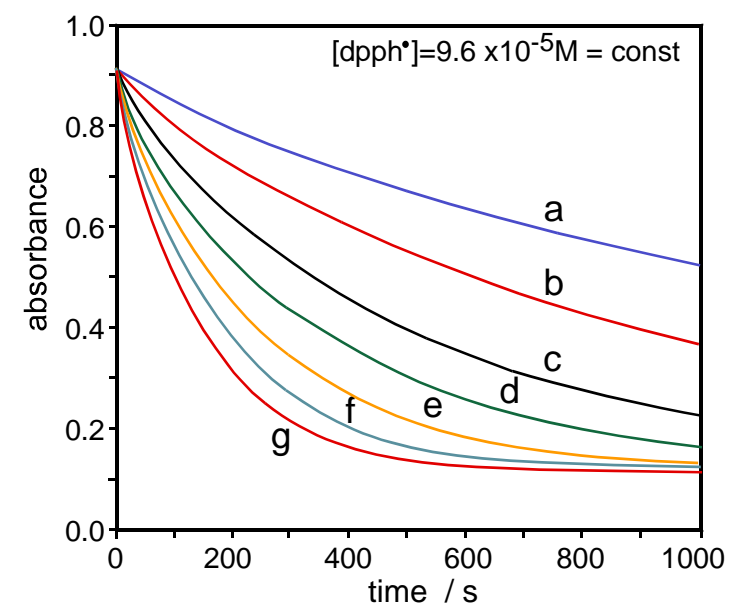

Figure S5. First order plots of decay of $\mathbf{d p p h}^{\bullet}\left(9.6 \times 10^{-5} \mathrm{M}\right)$ reacting with 2-tert-butyl4,6-dimethylphenol in acetone. Concentrations of $\mathrm{Bu}^{\mathrm{t}} \mathrm{Me}_{2}$-phenol are: a) $31.9 \mathrm{mM}, \mathrm{b}$ ) $24.8 \mathrm{mM}, \mathrm{c}) 19.3 \mathrm{mM}, \mathrm{d}) 14.5 \mathrm{mM}$, e) $10.9 \mathrm{mM}$, f) $6.5 \mathrm{mM}, \mathrm{g}) 3.9 \mathrm{mM}$. 


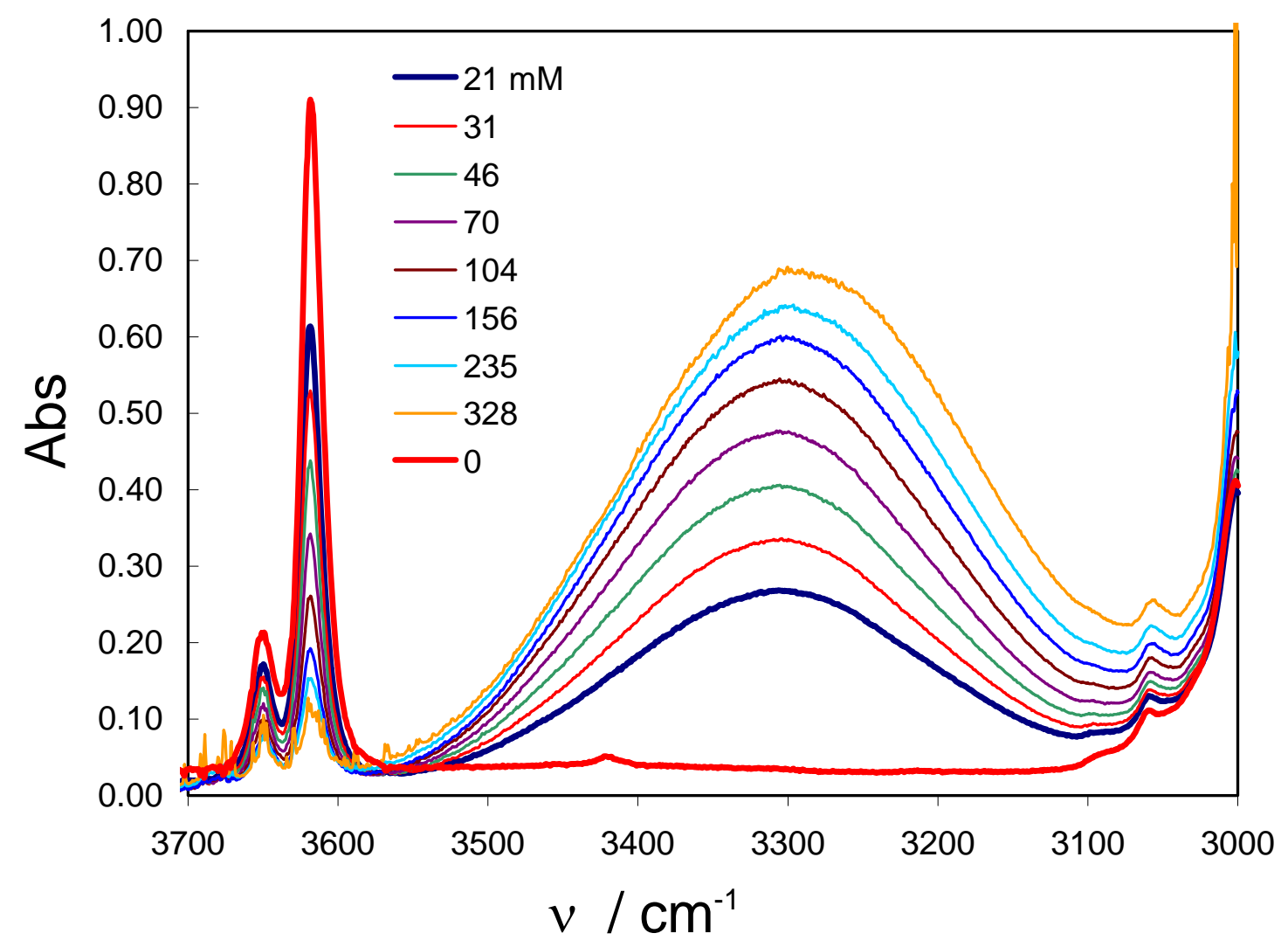

Figure S6. IR spectra of $22.4 \mathrm{mM}$ of 2-tert-butyl-4,6-dimethylphenol in $\mathrm{CCl}_{4}$ containing DMSO at concentrations ramging from 0 to $328 \mathrm{mM}$. The free $\mathrm{OH}$ stretching band having a maximum at $3619 \mathrm{~cm}^{-1}$ was used for calculation of the concentration of non-hydrogen bonded $\mathrm{Bu}^{\mathrm{t}} \mathrm{Me}_{2}$-phenol. 
Table S25. Parameters used for calculation of the equilibrium constant $K$ for HB complex formation between 2-tert-butyl-4,6-dimethylphenol and DMSO. Symbols are the same as in Figure S22. Plots of $[\mathrm{ArOH}]_{\mathrm{o}} /[\mathrm{ArOH}]_{\text {free }}$ vs. $[\mathrm{DMSO}]_{\text {free }}$ are presented in Figure S7. All concentrations in mM units.

\begin{tabular}{cccccc}
\hline$[\mathrm{ArOH}]_{\mathrm{o}}$ & {$[\mathrm{ArOH}]_{\text {free }}$} & {$[\mathrm{HB}]$} & {$[\mathrm{DMSO}]_{\mathrm{o}}$} & {$[\mathrm{DMSO}]_{\text {free }}$} & {$[\mathrm{ArOH}]_{\mathrm{o}} /[\mathrm{ArOH}]_{\text {free }}$} \\
\hline & \multicolumn{5}{c}{$\mathrm{Bu}^{\mathrm{t}} \mathrm{Me}_{2}$-phenol + DMSO } \\
22.35 & 22.35 & 0.0 & 0.0 & 0.0 & 1.00 \\
22.35 & 16.81 & 5.5 & 21.0 & 15.5 & 1.33 \\
22.35 & 14.86 & 7.5 & 31.0 & 23.5 & 1.50 \\
22.35 & 12.73 & 9.6 & 46.0 & 36.4 & 1.76 \\
22.35 & 10.42 & 11.9 & 70.0 & 58.1 & 2.14 \\
22.35 & 8.01 & 14.3 & 104.0 & 89.7 & 2.79 \\
22.35 & 5.95 & 16.4 & 156.0 & 139.6 & 3.76 \\
22.35 & 4.22 & 18.1 & 235.0 & 216.9 & 5.29 \\
22.35 & 3.23 & 19.1 & 328.0 & 308.9 & 6.91 \\
& & $\mathrm{Bu}^{\mathrm{t}} \mathrm{Me} \mathrm{e}_{2}$-phenol + DMSO & \\
18.22 & 18.22 & 0.0 & 0.0 & 0.0 & \\
18.22 & 14.22 & 4.0 & 21.0 & 17.0 & 1.00 \\
18.22 & 12.19 & 6.0 & 31.0 & 25.0 & 1.28 \\
18.22 & 9.97 & 8.2 & 46.0 & 37.8 & 1.49 \\
18.22 & 8.36 & 9.9 & 70.0 & 60.1 & 2.18 \\
18.22 & 6.24 & 12.0 & 104.0 & 92.0 & 2.92 \\
18.22 & 4.38 & 13.8 & 156.0 & 142.2 & 4.16 \\
18.22 & 3.19 & 15.0 & 235.0 & 220.0 & 5.70 \\
18.22 & 1.96 & 16.3 & 328.0 & 311.7 & 9.27 \\
\hline
\end{tabular}
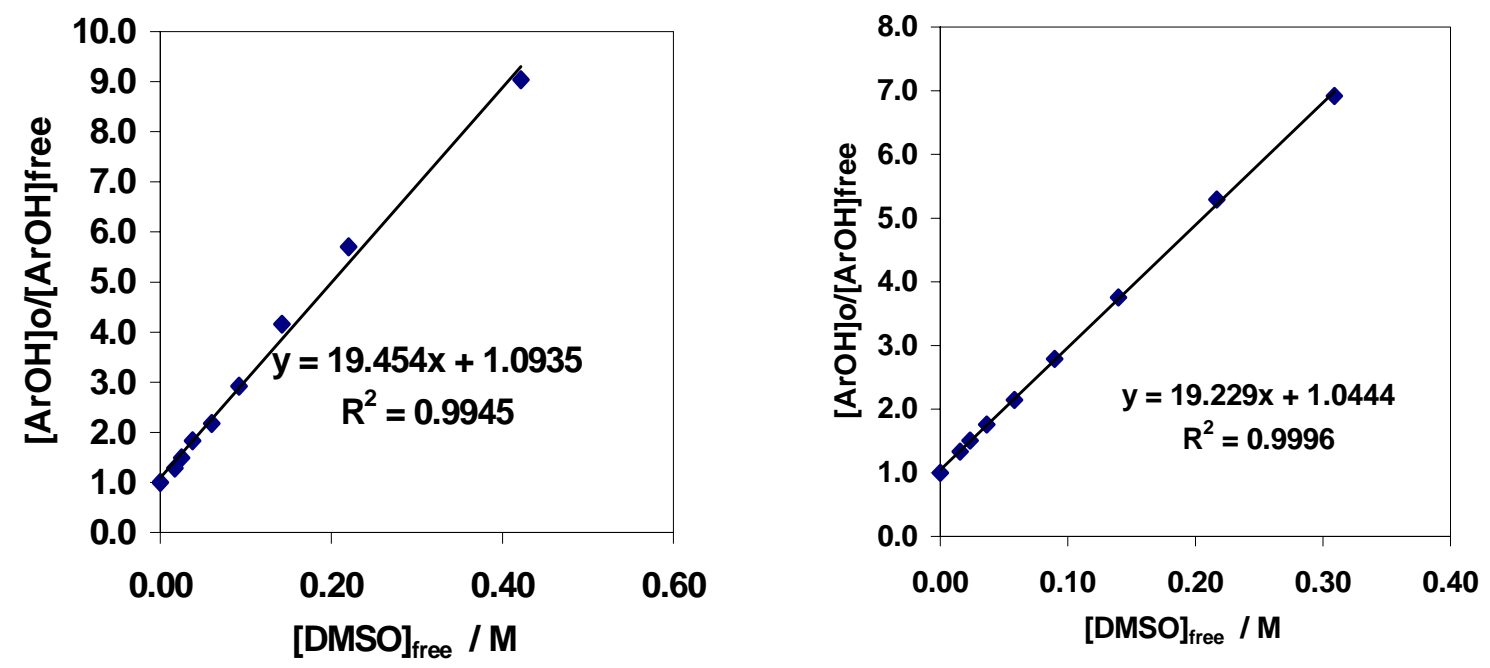

Figure S7. Plots of $[\mathrm{ArOH}]_{\mathrm{o}} /[\mathrm{ArOH}]_{\text {free }}$ vs. [DMSO $]_{\text {free }}$ for $\mathrm{Bu}^{\mathrm{t}} \mathrm{Me}_{2}$-phenol. The slopes of the straight lines yield the HB equilibrium constants. The mean value $(K=19.3)$ was used for calculation of $\log K_{A}^{H}=0.84$ (for DMSO $L_{B}=1.24$ and $D_{B}=0.266$, see ref. 10). The results yield $\alpha_{2}^{H}=0.42 \pm 0.01$ for $\mathrm{Bu}^{\mathrm{t}} \mathrm{Me}_{2}$-phenol. 

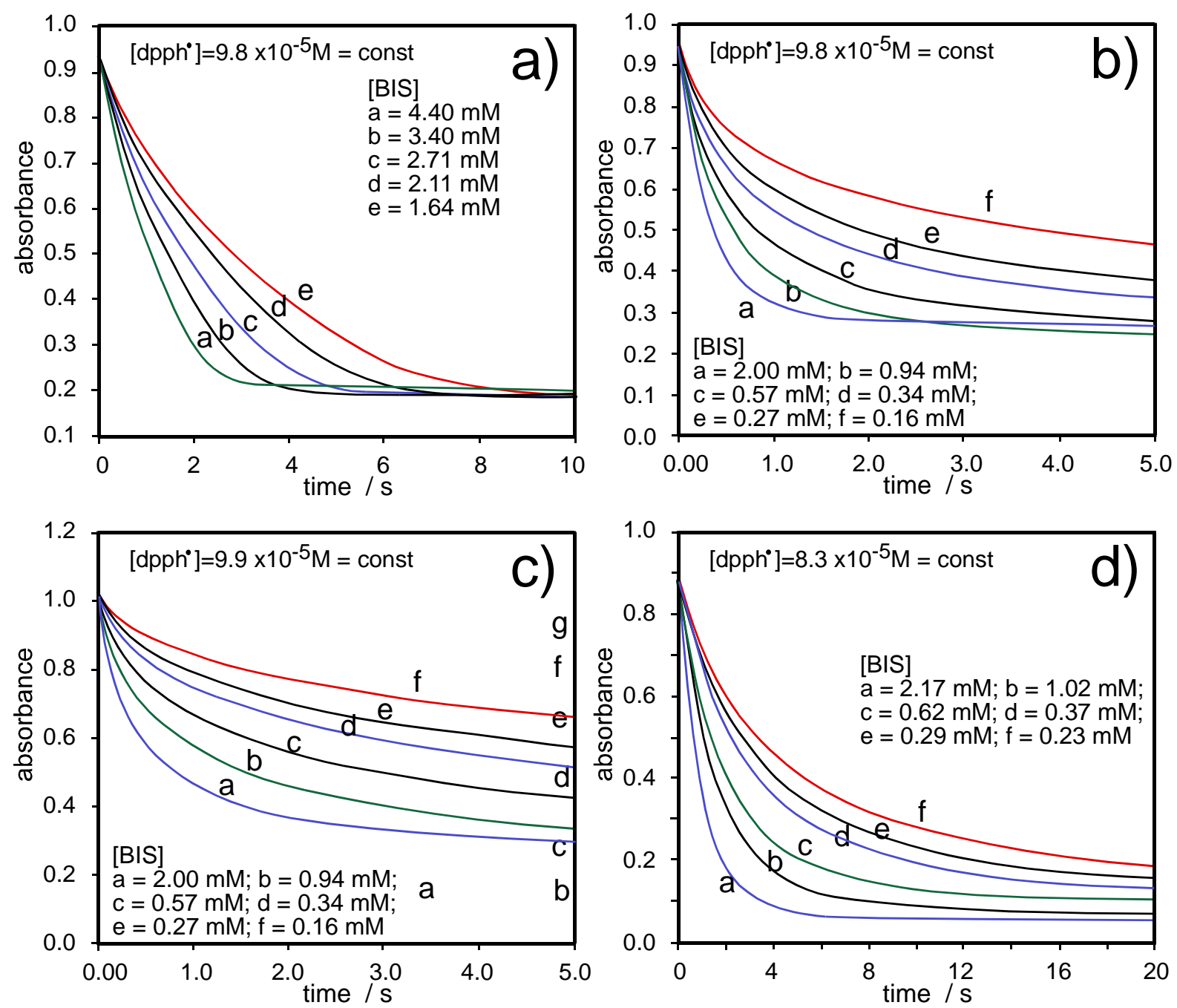

Figure S8. Kinetic traces of $\mathbf{d p p h}^{\bullet}$ decay in DMSO without acetic acid (Panel a), and in DMSO containing b) $10 \mathrm{mM}$, c) $100 \mathrm{mM}$ and d) $1000 \mathrm{mM}$ acetic acid. 


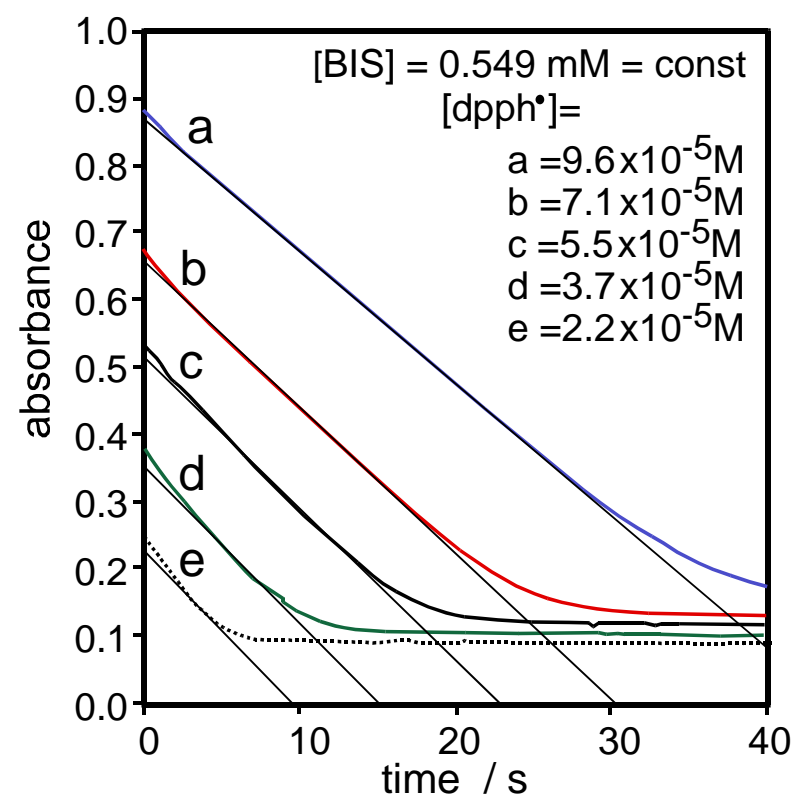

Figure S9. Zero-order plots of $\mathbf{d p p h}^{\bullet}$ decay in its reaction with BIS in acetone. The slopes of straight line sections were determined as: a) $-0.0196, b)-0.0214, c)-$ 0.0214, d) -0.023 , e) -0.0221 .
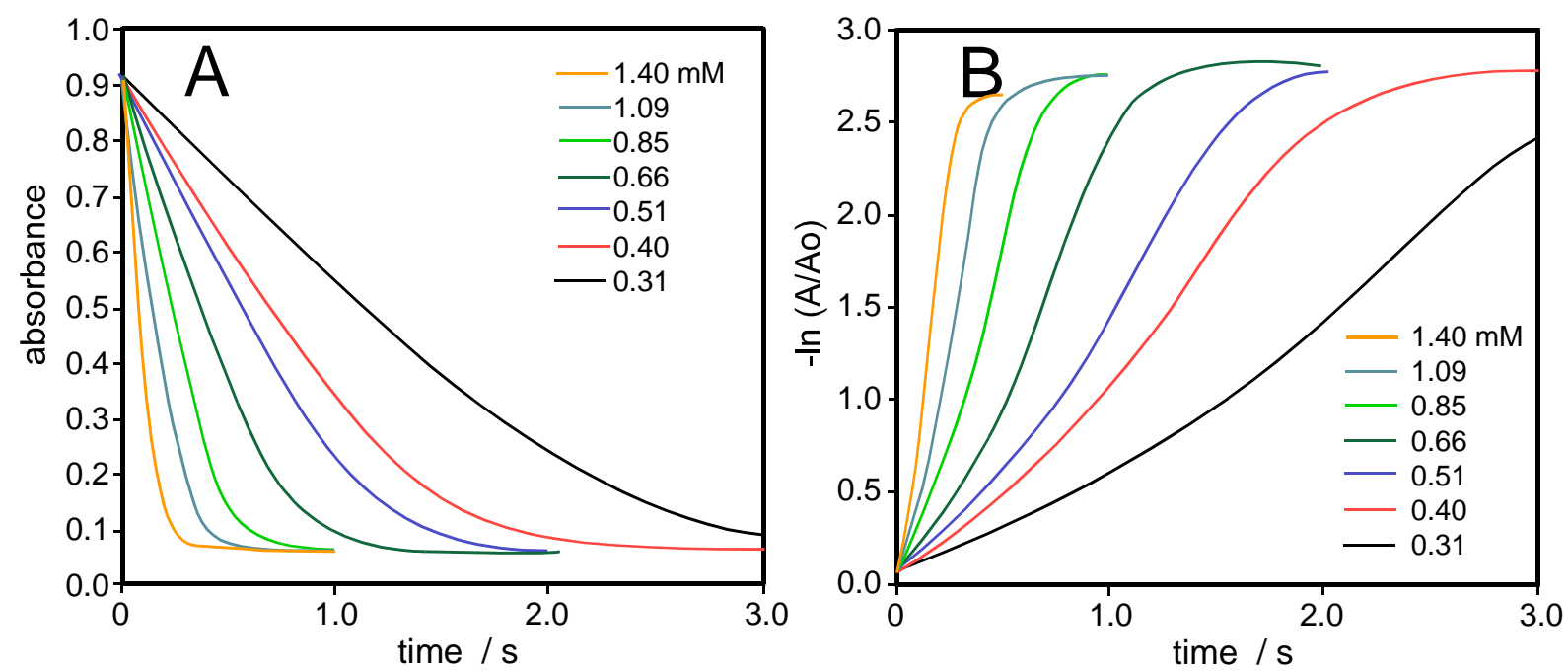

Figure S10. Panel A: Zero-order plots of $\mathbf{d p p h}^{\bullet}$ decay in its reaction with BIS in acetonitrile $\left(\left[\mathrm{dpph}^{\circ}\right]=8.9 \times 10^{-5} \mathrm{M}\right)$. Panel B: Plots of negative logarithms of normalized absorbance obtained from the kinetic curves in Panel $A$. 